\title{
Lyapunov, Bohl and Sacker-Sell Spectral Intervals for Differential-Algebraic Equations *
}

\author{
Vu Hoang Linh ${ }^{\dagger} \quad$ Volker Mehrmann ${ }^{\ddagger}$
}

November 8, 2008

\begin{abstract}
Lyapunov and exponential dichotomy spectral theory is extended from ordinary differential equations (ODEs) to nonautonomous differential-algebraic equations (DAEs). By using orthogonal changes of variables, the original DAE system is transformed into appropriate condensed forms, for which concepts such as Lyapunov exponents, Bohl exponents, exponential dichotomy and spectral intervals of various kinds can be analyzed via the resulting underlying ODE. Some essential differences between the spectral theory for ODEs and that for DAEs are pointed out. It is also discussed how numerical methods for computing the spectral intervals associated with Lyapunov and Sacker-Sell (exponential dichotomy) can be extended from those methods proposed for ODEs. Some numerical examples are presented to illustrate the theoretical results.
\end{abstract}

Keywords: differential-algebraic equations, strangeness index, Lyapunov exponent, Bohl exponent, Sacker-Sell spectrum, exponential dichotomy, spectral interval, smooth QR factorization, continuous $\mathrm{QR}$ algorithm, discrete $\mathrm{QR}$ algorithm, kinematic equivalence, Steklov function

AMS(MOS) subject classification: 65L07, 65L80, 34D08, 34D09

\section{Introduction}

More than a century ago, fundamental concepts and results for the stability theory of ordinary differential equations were presented in Lyapunov's famous thesis [59]. One of the most important notions, the so-called Lyapunov exponent (or Lyapunov characteristic number), has proved very useful in studying growth rates of solutions to linear ODEs. In the nonlinear case, by linearizing along a particular solution, Lyapunov exponents also give information about the convergence or divergence rates of nearby solutions. The spectral theory for ODEs was further developed throughout the 20th century, and concepts such as Bohl exponents, exponential dichotomy (also well-known as Sacker-Sell) spectra were introduced, see [1, 19, 20, 70]. Unlike the development of the analytic theory, the development of numerical methods to compute Lyapunov exponents and also other spectral intervals has only recently been studied. In a series of papers, see [23, 24, 26, 27, 29, 30, 31], Dieci and Van Vleck have developed algorithms for the computation of Lyapunov and Bohl exponents as well as Sacker-Sell spectral intervals. These methods have also been analyzed concerning their sensitivity under small perturbations (stability), the relationship between different spectra, the error analysis, and efficient implementation techniques.

\footnotetext{
${ }^{*}$ This research was supported by Deutsche Forschungsgemeinschaft, through MATHEON, the DFG Research Center "Mathematics for Key Technologies" in Berlin.

${ }^{\dagger}$ Faculty of Mathematics, Mechanics and Informatics, Vietnam National University, 334, Nguyen Trai Str., Thanh Xuan, Hanoi, Vietnam.

${ }^{\ddagger}$ Institut für Mathematik, MA 4-5, Technische Universität Berlin, D-10623 Berlin, Fed. Rep. Germany.
} 
This paper is devoted to the generalization of some theoretical results as well as numerical methods from the spectral theory for ODEs to differential-algebraic equations (DAEs). In particular, we are interested in the characterization of the dynamical behavior of solutions to initial value problems for linear systems of DAEs

$$
E(t) \dot{x}=A(t) x+f(t)
$$

on the half-line $\mathbb{I}=[0, \infty)$, together with an initial condition

$$
x(0)=x_{0} \text {. }
$$

Here we assume that $E, A \in C\left(\mathbb{I}, \mathbb{R}^{n \times n}\right)$, and $f \in C\left(\mathbb{I}, \mathbb{R}^{n}\right)$ are sufficiently smooth. We use the notation $C\left(\mathbb{I}, \mathbb{R}^{n \times n}\right)$ to denote the space of continuous functions from $\mathbb{I}$ to $\mathbb{R}^{n \times n}$.

Linear systems of the form (1) occur when one linearizes a general implicit nonlinear system of DAEs

$$
F(t, x, \dot{x})=0, \quad t \geq 0,
$$

along a particular solution [12]. In this paper for the discussion of spectral intervals, we restrict ourselves to regular DAEs, i. e., we require that (1) (or (3) locally) has a unique solution for sufficiently smooth $E, A, f(F)$ and appropriately chosen (consistent) initial conditions, see [50] for a discussion of existence and uniqueness of solution of more general nonregular DAEs.

DAEs like (1) and (3) arise in constrained multibody dynamics [36], electrical circuit simulation $[38,39]$, chemical engineering [32, 33] and many other applications, in particular when the dynamics of a system is constrained or when different physical models are coupled together in automatically generated models [64]. While DAEs provide a very convenient modeling concept, many numerical difficulties arise due to the fact that the dynamics is constrained to a manifold, which often is only given implicitly, see $[9,41,67]$ or the recent textbook [50]. These difficulties are typically characterized by one of many index concepts that exist for DAEs, see [9, 37, 41, 50].

The fact that the dynamics of DAEs is constrained also requires a modification of most classical concepts of the qualitative theory that was developed for ODEs. Different stability concepts for DAEs have been discussed already in $[2,42,43,53,60,62,68,69,71,72,73,74]$. Only very few papers, however, discuss the spectral theory for DAEs, see $[17,18]$ for results on Lyapunov exponents and Lyapunov regularity, [57] for the concept of exponential dichotomy used in numerical solution to boundary value problems, and [16, 35] for robustness results of exponential stability and Bohl exponents. All these papers use the tractability index approach as it was introduced in $[37,61]$ and consider linear systems of DAEs of tractability index 1 , only. Here we allow general regular DAEs of arbitrary index and we use reformulations based on derivative arrays as well as the strangeness index concept [50]. As in the ODE case there is also a close relation of the spectral theory to the theory of adjoint equations which has recently been studied in the context of control problems in $[4,5,6,14,51,52]$.

In this paper, we systematically extend the classical spectral concepts (Lyapunov, Bohl, SackerSell) that were introduced for ODEs, to general linear DAEs with variable coefficients of the form (1). We show that substantial differences in the theory arise and that most statements in the classical ODE theory hold for DAEs only under further restrictions, here our results extend results on asymptotic stability given in [53]. After deriving the concepts and analyzing the relationship between the different concepts of spectral intervals, we then derive two alternative numerical approaches to compute the corresponding spectra.

The outline of the paper is as follows. In the following section, we recall some concepts from the theory of differential-algebraic equations. We discuss in detail the extension of spectral concepts from ODEs to DAEs in Section 3. The relation between the spectral characteristics of DAE systems and those of their underlying ODE systems is investigated. Furthermore, the stability of the spectra with respect to perturbations arising in the system data is analyzed. In Section 4 we propose numerical methods for computing the Lyapunov and the Sacker-Sell (exponential dichotomy) spectral intervals and discuss implementation details as well as the associated error analysis. In Section 5 we present numerical examples to illustrate the theoretical results and the properties of the numerical methods. We finish the paper with a summary and a discussion of open problems. 


\section{A review of DAE theory}

In this section we briefly recall some concepts from the theory of differential-algebraic equations, see e.g. $[9,37,50,66]$. We follow [50] in notation and style of presentation.

Definition 1 Consider system (1) with sufficiently smooth coefficient functions $E, A$. A function $x: \mathbb{I} \rightarrow \mathbb{R}^{n}$ is called a solution of (1) if $x \in C^{1}\left(\mathbb{I}, \mathbb{R}^{n}\right)$ and $x$ satisfies (1) pointwise. It is called a solution of the initial value problem (1)-(2) if $x$ is a solution of (1) and satisfies (2). An initial condition (2) is called consistent if the corresponding initial value problem has at least one solution.

For the analysis as in $[11,13,48,50]$, we use derivative arrays

$$
M_{\ell}(t) \dot{z}_{\ell}=N_{\ell}(t) z_{\ell}+g_{\ell}(t)
$$

where

$$
\begin{aligned}
& \left(M_{\ell}\right)_{i, j}=\left(\begin{array}{c}
i \\
j
\end{array}\right) E^{(i-j)}-\left(\begin{array}{c}
i \\
j+1
\end{array}\right) A^{(i-j-1)}, i, j=0, \ldots, \ell, \\
& \left(N_{\ell}\right)_{i, j}= \begin{cases}A^{(i)} & \text { for } i=0, \ldots, \ell, j=0, \\
0 & \text { otherwise }\end{cases} \\
& \left(z_{\ell}\right)_{j}=x^{(j)}, j=0, \ldots, \ell, \\
& \left(g_{\ell}\right)_{i}=f^{(i)}, i=0, \ldots, \ell,
\end{aligned}
$$

using the convention that $\left(\begin{array}{l}i \\ j\end{array}\right)=0$ for $i<0, j<0$ or $j>i$. In more detail, we have

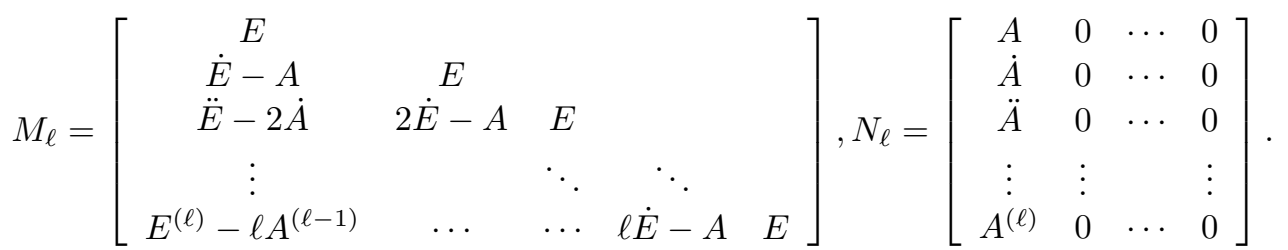

To guarantee existence and uniqueness of solutions, we make the following hypothesis, see [50].

Hypothesis 2 There exist integers $\mu$, a, and $d$ such that the inflated pair $\left(M_{\mu}, N_{\mu}\right)$ associated with the given pair of matrix functions $(E, A)$ has the following properties:

1. For all $t \in \mathbb{I}$ we have $\operatorname{rank} M_{\mu}(t)=(\mu+1) n-$ a such that there exists a smooth matrix function $Z_{2}$ of size $(\mu+1) n \times a$ and pointwise maximal rank satisfying $Z_{2}^{T} M_{\mu}=0$.

2. For all $t \in \mathbb{I}$ we have $\operatorname{rank} \hat{A}_{2}(t)=a$, where $\hat{A}_{2}=Z_{2}^{T} N_{\mu}\left[I_{n} 0 \cdots 0\right]^{T}$ such that there exists a smooth matrix function $T_{2}$ of size $n \times d, d=n-a$, and pointwise maximal rank satisfying $\hat{A}_{2} T_{2}=0$.

3. For all $t \in \mathbb{I}$ we have rank $E(t) T_{2}(t)=d$ such that there exists a smooth matrix function $Z_{1}$ of size $n \times d$ and pointwise maximal rank satisfying $\operatorname{rank} \hat{E}_{1} T_{2}=d$ with $\hat{E}_{1}=Z_{1}^{T} E$.

Since Gram-Schmidt orthonormalization is a continuous process, we may assume without loss of generality that the columns of the matrix functions $Z_{1}, Z_{2}$, and $T_{2}$ in Hypothesis 2 are pointwise orthonormal.

Definition 3 The smallest possible $\mu$ for which Hypothesis 2 holds is called the strangeness index of (1). Systems with vanishing strangeness index are called strangeness-free.

The strangeness index can be considered as a generalization of the differentiation index as introduced in [8], see [50] for a detailed analysis of the relationship between different index concepts. It has been shown in [47], see also [50], that under some constant rank conditions, every uniquely solvable (regular) linear DAE of the form (1) with sufficiently smooth $E, A$ satisfies Hypothesis 2 and that there exists a reduced system

$$
\hat{E}(t) \dot{x}=\hat{A}(t) x+\hat{f}(t)
$$


that is strangeness-free and has the same solution as (1), where

$$
\hat{E}(t)=\left[\begin{array}{c}
\hat{E}_{1}(t) \\
0
\end{array}\right], \hat{A}=\left[\begin{array}{c}
\hat{A}_{1} \\
\hat{A}_{2}
\end{array}\right],
$$

with block entries

$$
\hat{E}_{1}=Z_{1}^{T} E, \quad \hat{A}_{1}=Z_{1}^{T} A, \quad \hat{A}_{2}=Z_{2}^{T} N_{\mu}\left[\begin{array}{llll}
I_{n} & 0 & \cdots & 0
\end{array}\right]^{T} .
$$

System (7) can be viewed as a different representation (remodeling) of system (1), where all necessary differentiations of (1) that are needed to describe the solution are already represented in the model. This representation avoids many of the numerical difficulties that are associated with DAEs that have a non-vanishing strangeness-index (differentiation index larger than 1), see [9, 50]. The reduction to the form $(7)$ can be carried out in a numerically stable way at any time instance $t$, see $[55,50]$ and this idea can also be extended to over- and underdetermined systems as well as locally to general nonlinear systems $[54,49,50]$. For this reason, in the following, we assume that the DAE is given in the form (7) and for ease of notation we leave off the hats. Furthermore, a matrix function will be said nonsingular (orthogonal) if it is pointwise nonsingular (orthogonal).

\section{Spectral theory for DAEs}

In this section we generalize the classical spectral results for ODEs to DAEs. We refer to [26, 27, $30,44]$ or [58] for more details on the theory for ODEs. An essential step in the computation of spectral intervals for linear DAEs of the form (1) is to first transform the system to a reduced strangeness-free form (7), which has the same solution set as (1), see [50], and then to consider the spectral results in this framework. This transformation will not alter the spectral sets which will be defined in terms of the fundamental solution matrices that have not changed. Under Hypothesis 2 this transformation can always be done and this reduced form can even be computed numerically at every time instance $t$. For this reason, we may assume in the following that the system is given in the reduced form (7), i.e. we assume that our homogeneous DAE is already strangeness-free and has the form

$$
E(t) \dot{x}=A(t) x, \quad t \in \mathbb{I},
$$

where

$$
E(t)=\left[\begin{array}{c}
E_{1}(t) \\
0
\end{array}\right], A(t)=\left[\begin{array}{c}
A_{1}(t) \\
A_{2}(t)
\end{array}\right],
$$

and $E_{1} \in C\left(\mathbb{I}, \mathbb{R}^{d \times n}\right)$ and $A_{2} \in C\left(\mathbb{I}, \mathbb{R}^{(n-d) \times n}\right)$ are of full column rank.

\subsection{Lyapunov exponents and Lyapunov spectral intervals}

We first discuss the concepts of Lyapunov exponents and Lyapunov spectral intervals.

Definition 4 A matrix function $X \in C^{1}\left(\mathbb{I}, \mathbb{R}^{n \times k}\right), \quad d \leq k \leq n$, is called fundamental solution matrix of (9) if each of its columns is a solution to (9) and $\operatorname{rank} X(t)=d$, for all $t \geq 0$.

A fundamental solution matrix is said to be maximal if $k=n$ and minimal if $k=d$, respectively. A maximal fundamental matrix solution, denoted by $X(t, s)$, is called principal if it satisfies the projected initial condition $E\left(t_{0}\right)\left(X\left(t_{0}, t_{0}\right)-I\right)=0$, for some $t_{0} \geq 0$.

A major difference between ODEs and DAEs is that fundamental solution matrices for DAEs are not necessarily square and of full-rank. Every fundamental solution matrix has exactly $d$ linearly independent columns and a minimal fundamental matrix solution can be easily made maximal by adding $n-d$ zero columns. 
Definition 5 For a given fundamental solution matrix $X$ of a strangeness-free DAE system of the form (9), and for $d \leq k \leq n$, we introduce

$$
\lambda_{i}^{u}=\limsup _{t \rightarrow \infty} \frac{1}{t} \ln \left\|X(t) e_{i}\right\| \quad \text { and } \lambda_{i}^{\ell}=\liminf _{t \rightarrow \infty} \frac{1}{t} \ln \left\|X(t) e_{i}\right\|, \quad i=1,2, \ldots, k,
$$

where $e_{i}$ denotes the $i$-th unit vector. The columns of a minimal fundamental solution matrix form a normal basis if $\Sigma_{i=1}^{d} \lambda_{i}^{u}$ is minimal. The $\lambda_{i}^{u}, i=1,2, \ldots, d$, belonging to a normal basis are called (upper) Lyapunov exponents and the intervals $\left[\lambda_{i}^{\ell}, \lambda_{i}^{u}\right], i=1,2, \ldots, d$, are called Lyapunov spectral intervals. The set of the Lyapunov spectral intervals is called the Lyapunov spectrum of (9).

Definition 6 Suppose that $U \in C\left(\mathbb{I}, \mathbb{R}^{n \times n}\right)$ and $V \in C^{1}\left(\mathbb{I}, \mathbb{R}^{n \times n}\right)$ are nonsingular matrix functions such that $V$ and $V^{-1}$ are bounded. Then the transformed DAE system

$$
\tilde{E}(t) \dot{\tilde{x}}=\tilde{A}(t) \tilde{x}
$$

with $\tilde{E}=U E V, \tilde{A}=U A V-U E \dot{V}$ and $x=V \tilde{x}$ is called globally kinematically equivalent to (9) and the transformation is called a global kinematical equivalence transformation. If $U \in$ $C^{1}\left(\mathbb{I}, \mathbb{R}^{n \times n}\right)$ and, furthermore, also $U$ and $U^{-1}$ are bounded then we call this a strong global kinematical equivalence transformation.

It is clear that the Lyapunov exponents of a DAE system as well as the normality of a basis formed by the columns of a fundamental solution matrix are preserved under global kinematic equivalence transformations.

Lemma 7 Consider a strangeness-free DAE system of the form (9) with continuous coefficients and a minimal fundamental solution matrix $X$. Then there exist orthogonal matrix functions $U \in C\left(\mathbb{I}, \mathbb{R}^{n \times n}\right)$ and $V \in C^{1}\left(\mathbb{I}, \mathbb{R}^{n \times n}\right)$ such that in the fundamental matrix equation $E \dot{X}=A X$ associated with (9), the change of variables $X=V R$, with $R=\left[\begin{array}{c}R_{1} \\ 0\end{array}\right]$ and $R_{1} \in C^{1}\left(\mathbb{I}, \mathbb{R}^{d \times d}\right)$, and the multiplication of both sides of the system from the left with $U^{T}$ leads to the system

$$
\mathcal{E}_{1} \dot{R}_{1}=\mathcal{A}_{1} R_{1}
$$

where $\mathcal{E}_{1}:=U_{1}^{T} E V_{1}$ is nonsingular and $\mathcal{A}_{1}:=U_{1}^{T} A V_{1}-U_{1}^{T} E \dot{V}_{1}$. Here, $U_{1}$, $V_{1}$ are the matrix functions consisting of the first $d$ columns of $U, V$, respectively.

Proof. Since a smooth and full column rank matrix function has a smooth $Q R$-decomposition, see [25, Prop. 2.3], there exists an orthogonal matrix function $V$ such that $X=V R=\left[\begin{array}{c}R_{1} \\ 0\end{array}\right]$, where $R_{1}$ is nonsingular. By substituting $X=V R$ into the fundamental matrix equation $E \dot{X}=A X$, we obtain

$$
E V\left[\begin{array}{c}
\dot{R}_{1} \\
0
\end{array}\right]=(A V-E \dot{V})\left[\begin{array}{c}
R_{1} \\
0
\end{array}\right]
$$

Since, by assumption, the first $d$ rows of $E$ are of full row rank, we have that the first $d$ columns of $E V$, given by $E V_{1}$, have full column rank. Thus, there exists a smooth $Q R$-decomposition

$$
E V_{1}=U\left[\begin{array}{c}
\mathcal{E}_{1} \\
0
\end{array}\right]
$$

where $U$ is orthogonal and $\mathcal{E}_{1}$ is nonsingular. Looking at the leading $d \times d$ block in the transformed equation, we arrive at

$$
\mathcal{E}_{1} \dot{R}_{1}=\left[U_{1}^{T} A V_{1}-U_{1}^{T} E \dot{V}_{1}\right] R_{1},
$$

which proves the assertion.

The system (11) is an implicitly given ODE, since $\mathcal{E}_{1}$ is nonsingular. It is called essentially underlying implicit ODE system of (9). Since orthonormal changes of basis keep the Euclidean norm invariant, the Lyapunov exponents of the columns of the matrices $X$ and $R$, and therefore those of the two systems are the same. 
Theorem 8 Let $Z$ be a minimal fundamental solution matrix for (9) such that the upper Lyapunov exponents of its columns are ordered decreasingly. Then there exists a nonsingular upper triangular matrix $C \in \mathbb{R}^{d \times d}$ such that the columns of $X(\cdot)=Z(\cdot)$ C form a normal basis.

Proof. By Lemma 7, there exists an orthogonal matrix function $V$ such that $V^{T} Z=\left[\begin{array}{c}R_{1} \\ 0\end{array}\right]$ with $R_{1}$ satisfying the implicit system

$$
\mathcal{E}_{1} \dot{R}_{1}=\mathcal{A}_{1} R_{1}
$$

or equivalently, satisfying the explicit ODE system

$$
\dot{R}_{1}=\mathcal{E}_{1}^{-1} \mathcal{A}_{1} R_{1}
$$

Here $\mathcal{E}_{1}, \mathcal{A}_{1}$ are defined as in Lemma 7 . Note that the Lyapunov exponents of $Z$ are exactly the Lyapunov exponents of $R_{1}$. Due to Lyapunov's theorem on the construction of a normal basis for ODEs (see [59]), there exists an upper triangular nonsingular matrix $C \in \mathbb{R}^{d \times d}$ such that the columns of $R_{1} C$ form a normal basis of (11). This implies that the columns of $R C=V^{T} Z C$ form a normal basis as well. Because the normality is preserved under global kinematical equivalence transformations, the proof is complete.

As in the case of ODEs it is useful to introduce the adjoint equation to (9), see also $[14,5,51,52]$.

Definition 9 The DAE system

$$
\frac{d}{d t}\left(E^{T} y\right)=-A^{T} y, \quad \text { or } \quad E^{T}(t) \dot{y}=-\left[A^{T}(t)+\dot{E}^{T}(t)\right] y, \quad t \in \mathbb{I},
$$

is called the adjoint system associated with (9).

Lemma 10 Fundamental solution matrices $X, Y$ of (9) and its adjoint equation (12) satisfy the Lagrange identity

$$
Y^{T}(t) E(t) X(t)=Y^{T}(0) E(0) X(0), t \in \mathbb{I} .
$$

Let $U, V \in C^{1}\left(\mathbb{I}, \mathbb{R}^{n \times n}\right)$ define a strong global kinematic equivalence for system (9). Then the adjoint of the transformed DAE system (10) is strongly globally kinematically equivalent to the adjoint of (9).

Proof. Differentiating the product $Y(t)^{T} E(t) X(t)$ and using the definition of the adjoint equation, we obtain (leaving off the arguments) that

$$
\frac{d}{d t}\left(Y^{T} E\right) X+Y^{T} E \dot{X}=-Y^{T} A X+Y^{T} A X=0
$$

and hence the Lagrange identity follows. By assumption, the matrices $V^{T}, U^{T}$ define a strong global kinematic equivalence transformation for the adjoint equation leading to the adjoint of (10).

Remark 11 In the ODE theory, the adjoint equations are easily derived from the Lagrange identity. Nevertheless for DAEs, since a fundamental matrix solution is not necessarily square or may be singular, the Lagrange identity does not imply the ajoint system (12). The concept of adjoint is defined only for some classes of DAEs. That is, given a DAE, it may happen that its adjoint DAE does not exist or sometimes it is not clear at all what is an adjoint system. For more details on adjoint DAEs, see [5, 14] and references therein.

The relationship between the dynamics of a DAE system and its adjoint is more complicated than in the ODE case, except if some extra assumptions are added. In order to see this and to better understand the dynamical behavior of DAEs, we apply an orthogonal change of basis to transform the system (9) into appropriate condensed forms. 
Theorem 12 Consider the strangeness-free DAE system (9). If the pair of coefficient matrices is sufficiently smooth, then there exists an orthogonal matrix function $\hat{Q} \in C^{1}\left(\mathbb{I}, \mathbb{R}^{n \times n}\right)$ such that by the change of variables $\hat{x}=\hat{Q}^{T} x$, the submatrix $E_{1}$ is compressed, i.e., the transformed system has the form

$$
\left[\begin{array}{cc}
\hat{E}_{11} & 0 \\
0 & 0
\end{array}\right] \dot{\hat{x}}=\left[\begin{array}{cc}
\hat{A}_{11} & \hat{A}_{12} \\
\hat{A}_{21} & \hat{A}_{22}
\end{array}\right] \hat{x}, \quad t \in \mathbb{I} .
$$

Furthermore, the system (13) is still strangeness-free and thus $\hat{E}_{11}$ and $\hat{A}_{22}$ are nonsingular.

Proof. In order to show the existence of appropriate transformations, we use again the theorem on the existence of smooth $Q R$ decompositions, see [21, Prop. 2.3] and [50, Thm. 3.9]. If $E$ is continuously differentiable, then there exist a matrix function $\hat{Q}_{1} \in C^{1}\left(\mathbb{I}, \mathbb{R}^{n \times d}\right)$ with orthonormal columns and a nonsingular $\hat{E}_{11} \in C^{1}\left(\mathbb{I}, \mathbb{R}^{d \times d}\right)$ such that

$$
E_{1}=\hat{E}_{11} \hat{Q}_{1}^{T} \text {. }
$$

Since $d$ rows of $\hat{Q}_{1}^{T}$ pointwise form orthonormal basis in $\mathbb{R}^{n}$ and since the Gram-Schmidt process is continuous, we can complete this basis by adding a smooth (and pointwise orthonormal) matrix $\hat{Q}_{2} \in C^{1}\left(\mathbb{I}, \mathbb{R}^{n \times(n-d)}\right)$ so that

$$
\hat{Q}:=\left[\begin{array}{ll}
\hat{Q}_{1} & \hat{Q}_{2}
\end{array}\right]
$$

is pointwise orthogonal. Then, we have

$$
E_{1}=\left[\begin{array}{ll}
\hat{E}_{11} & 0
\end{array}\right] \hat{Q}^{T} .
$$

Since we have started with a strangeness-free system, it follows that the corresponding transformed matrix $\hat{A}$ partitioned as in (13) has a nonsingular block $\hat{A}_{22}$.

Remark 13 Alternatively we could have used a transformation in Theorem 12 that compresses the block $A_{2}$, thus obtaining a transformed system

$$
\left[\begin{array}{cc}
\tilde{E}_{11} & \tilde{E}_{12} \\
0 & 0
\end{array}\right] \dot{\tilde{x}}=\left[\begin{array}{cc}
\tilde{A}_{11} & \tilde{A}_{12} \\
0 & \tilde{A}_{22}
\end{array}\right] \tilde{x}, \quad t \in \mathbb{I} .
$$

with $\tilde{E}_{11}$ and $\tilde{A}_{22}$ nonsingular. The proof for the condensed form (14) follows analogously to that of Theorem 12 by compressing the second block row of $A$, see also [15, Corollary 2.5]. Most of the results that we present below carry over directly to this system. Due to the use of orthogonal transformations, it is also clear that the two transformed systems (13) and (14) are globally kinematically equivalent. It is important to note in addition that the form (13) generalizes the semi-explicit form which appears frequently in applications, see [9]. So all the theoretical results derived for (13) apply directly to the class of semi-exlicit DAEs. In this case, all conditions can be checked directly for the original system. However, for numerical computations, the form (14) is more convenient. To calculate spectral intervals efficiently, we prefer transforming the DAE of general form (1) or (9) into the form (14) rather than (13).

System (13) is a strangeness-free DAE in semi-implicit form. Since $\hat{Q}$ is orthogonal and since the Euclidean norm is used, it follows that $\|\hat{x}\|=\|x\|$. Performing this transformations allows to separate the differential and the algebraic components of the solutions. Partitioning $\hat{x}=\left[\hat{x}_{1}^{T}, \hat{x}_{2}^{T}\right]^{T}$ appropriately, solving for the second component and substituting it into the first block equation one gets the associated underlying (implicit) $O D E$,

$$
\hat{E}_{11} \dot{\hat{x}}_{1}=\hat{A}_{s} \hat{x}_{1}
$$

where $\hat{A}_{s}:=\hat{A}_{11}-\hat{A}_{12} \hat{A}_{22}^{-1} \hat{A}_{21}$ denotes the Schur complement. For (14), the associated underlying implicit ODE system is

$$
\tilde{E}_{11} \tilde{x}_{1}=\tilde{A}_{11} \tilde{x}_{1},
$$

respectively. The following result extends the asymptotic stability results of [53] in terms of Lyapunov exponents. 
Theorem 14 Let $\lambda^{u}\left(\hat{A}_{22}^{-1} \hat{A}_{21}\right)$ be the upper Lyapunov exponent of the matrix function $\hat{A}_{22}^{-1} \hat{A}_{21}$. If

$$
\lambda^{u}\left(\hat{A}_{22}^{-1} \hat{A}_{21}\right) \leq 0
$$

then the (upper and lower) Lyapunov exponents of (13) and those of (15) coincide if they are both ordered decreasingly.

Proof. It is clear that each minimal fundamental solution matrix $\hat{X}$ of (13) has the form

$$
\hat{X}=\left[\begin{array}{c}
\hat{X}_{1} \\
-\hat{A}_{22}^{-1} \hat{A}_{21} \hat{X}_{1}
\end{array}\right],
$$

where $\hat{X}_{1}$ is a fundamental solution of (15). Let $\hat{x}$ be a column of $\hat{X}$. Then

$$
\hat{x}=\left[\begin{array}{c}
\hat{x}_{1} \\
-\hat{A}_{22}^{-1} \hat{A}_{21} \hat{x}_{1}
\end{array}\right],
$$

where $\hat{x}_{1}$ is the corresponding column of $\hat{X}_{1}$. Using the triangle inequality, we then have

$$
\left\|\hat{x}_{1}\right\| \leq\|\hat{x}\| \leq\left(1+\left\|\hat{A}_{22}^{-1} \hat{A}_{21}\right\|\right)\left\|\hat{x}_{1}\right\|
$$

from which it follows that

$$
\lambda^{u}\left(\hat{x}_{1}\right) \leq \lambda^{u}(\hat{x}) \leq \lambda^{u}\left(1+\left\|\hat{A}_{22}^{-1} \hat{A}_{21}\right\|\right)+\lambda^{u}\left(\hat{x}_{1}\right)=\lambda^{u}\left(\hat{x}_{1}\right)
$$

and thus, $\lambda^{u}(\hat{x})=\lambda^{u}\left(\hat{x}_{1}\right)$. Analogously we prove that $\lambda^{l}\left(\hat{x}_{1}\right) \leq \lambda^{l}(\hat{x})$.

Since $\lambda^{u}\left(\hat{A}_{22}^{-1} \hat{A}_{21}\right) \leq 0$, for any $\varepsilon>0$, there exists $T \geq 0$ such that

$$
\frac{1}{t} \ln \left(1+\left\|\hat{A}_{22}^{-1} \hat{A}_{21}\right\|\right) \leq \varepsilon \text { for all } t \geq T,
$$

which implies that $1+\left\|\hat{A}_{22}^{-1} \hat{A}_{21}\right\| \leq e^{\varepsilon t}, \quad$ for all $t \geq T$. As in the case of upper exponents, we have

$$
\|\hat{x}(t)\| \leq \sqrt{2} e^{\varepsilon t}\left\|\hat{x}_{1}(t)\right\|, \quad t \geq T .
$$

Hence, we obtain that $\lambda^{l}(\hat{x}) \leq \varepsilon+\lambda^{l}\left(\hat{x}_{1}\right)$. Since $\varepsilon$ can be chosen arbitrarily, it follows that $\lambda^{l}(\hat{x}) \leq \lambda^{l}\left(\hat{x}_{1}\right)$. Thus, it follows that $\lambda^{l}\left(\hat{x}_{1}\right)=\lambda^{l}(\hat{x})$. As a consequence of this construction, the columns of the fundamental solution matrix $\hat{X}$ of (13) form a normal basis if and only if the corresponding columns of $X_{1}$ form a normal basis of (15).

Remark 15 Assumption (17) ensures that the "algebraic" variable $\hat{x}_{2}$ cannot grow exponentially faster than the "differential" variable $\hat{x}_{1}$. Thus, the dynamics of the underlying ODE (15) essentially determines the dynamics of the DAE (13), see also [53]. A sufficient condition for (17) is that $\hat{A}_{22}^{-1} \hat{A}_{21}$ is bounded or has a less than exponential growth rate. This is for example the case if there exist constants $\gamma>0$ and $k \in \mathbb{N}$ such that $\left\|\hat{A}_{22}^{-1} \hat{A}_{21}(t)\right\| \leq \gamma t^{k}$ for all $t \in \mathbb{I}$.

Remark 16 Alternatively, we could use (14) and the corresponding underlying ODE (16). It is easy to prove the equality for the Lyapunov exponents of (14) and those of (16). In this case such a boundedness or restriction in the growth rate like (17) is not required. However, a similar boundedness condition on $\tilde{A}_{22}^{-1}$ in (14) will be needed, if one considers the analysis of perturbed or inhomogeneous DAE systems.

The next step of our analysis is the extension of the concept of Lyapunov-regularity to DAEs.

Definition 17 The DAE system (9) is said to be Lyapunov-regular if each of its Lyapunov spectral intervals reduces to a point, i.e., $\lambda_{i}^{l}=\lambda_{i}^{u}, i=1,2, \ldots, d$. 
To analyze the Lyapunov-regularity of the DAE system (9), we again study the transformed semi-implicit system (13) and the underlying ODE system. Since the Lyapunov exponents for a DAE system are preserved under global kinematic equivalence transformations, also the Lyapunovregularity is preserved, i. e. the DAE system (9) is Lyapunov-regular if and only if the semi-implicit DAE system (13) is Lyapunov-regular. Thus, we immediately have the following equivalence result.

Proposition 18 Consider the DAE system (13) and suppose that the boundedness condition (17) holds. Then, the DAE system (13) is Lyapunov-regular if and only if the underlying ODE system (15) is Lyapunov-regular.

Unlike for ODEs, to obtain the equivalence between the Lyapunov-regularity of (9) and that of its adjoint system we need some extra conditions.

Theorem 19 Consider the DAE system (13) and suppose that the boundedness condition (17) holds. Assume further, that for the transformed system (13) the conditions

$$
\lambda^{u}\left(\hat{A}_{12} \hat{A}_{22}^{-1}\right) \leq 0, \quad \lambda^{u}\left(\hat{E}_{11}\right) \leq 0, \quad \lambda^{u}\left(\hat{E}_{11}^{-1}\right) \leq 0
$$

hold. If $\lambda_{i}^{l}$ are the lower Lyapunov exponents order of (9) and - $\mu_{i}^{u}$ are the upper Lyapunov exponents of the adjoint system (12), both in increasing order, then

$$
\lambda_{i}^{l}=\mu_{i}^{u}, \quad i=1,2, \ldots, d,
$$

Furthermore, system (9) is regular if and only if (12) is regular, and in this case we have the Perron identity

$$
\lambda_{i}=\mu_{i}, \quad i=1,2, \ldots, d,
$$

where $\left\{-\mu_{i}\right\}_{i=1}^{d}$ are the Lyapunov exponents of (12) in increasing order.

Proof. Without loss of generality, we may consider the adjoint system (12) for the semi-implicit form (13)

$$
\left[\begin{array}{cc}
\hat{E}_{11}^{T} & 0 \\
0 & 0
\end{array}\right] \dot{\hat{y}}=-\left(\left[\begin{array}{cc}
\hat{A}_{11}^{T}+\hat{E}_{11}^{T} & \hat{A}_{21}^{T} \\
\hat{A}_{12}^{T} & \hat{A}_{22}^{T}
\end{array}\right]\right) \hat{y} .
$$

The underlying ODE of the adjoint system is then given by

$$
\hat{E}_{11}^{T} \dot{\hat{y}}_{1}=-\left(\hat{A}_{11}^{T}-\hat{A}_{21}^{T} \hat{A}_{22}^{-T} \hat{A}_{12}^{T}+\dot{\hat{E}}_{11}^{T}\right) \hat{y}_{1},
$$

which is exactly the adjoint of the underlying ODE system (15). It also follows immediately that the Lagrange identity $\hat{Y}_{1}^{T} \hat{E}_{11} \hat{X}_{1} \equiv$ const holds for fundamental matrix solutions $\hat{X}_{1}, \hat{Y}_{1}$ of $(15)$ and its adjoint, respectively. Note that if the columns of $X_{1}$ form a normal basis for (15) then those of $\hat{Y}_{1}:=\hat{E}_{11}^{-T} \hat{X}_{1}^{-T}$ form a normal basis for the adjoint. Hence, (20) holds for the Lyapunov spectra of the underlying ODE systems and due to the preservation of Lyapunov spectra under global kinematic equivalence, the proof is complete.

Corollary 20 Suppose that (9) is Lyapunov-regular and the assumptions of Theorem 19 hold. Then, with $\hat{A}_{s}$ defined as in (15), the limit

$$
\lim _{t \rightarrow \infty} \frac{1}{t} \int_{0}^{t} \operatorname{tr}\left(\hat{E}_{11}^{-1} \hat{A}_{s}(s)\right) d s
$$

exists and is equal to $\sum_{i=1}^{d} \lambda_{i}$.

Remark 21 The last two inequalities in (19) just imply $\lambda^{u}\left(\hat{E}_{11}\right)=\lambda^{u}\left(\hat{E}_{11}^{-1}\right)=0$. It is also important to note that the relation between the spectral intervals of (9) and its adjoint (12) stated in Theorem 19 is invariant under strongly global kinematic equivalence transformations. 
However, a global kinematic equivalence transformation that is not strong, may destroy the Perron identity as the following simple example demonstrates: Consider a scalar ODE and a kinematically equivalent implicit equation

$$
\dot{x}=-x, \quad \text { and } e^{t} \dot{x}=-e^{t} x .
$$

It is easy to check that the Perron identity holds for the first equation but this is not true for the second one.

The following examples show that, if one of the conditions (17), (19) fails to hold, then the DAE and its adjoint may be Lyapunov-regular, but the Perron identity (20) does not hold and also the converse implication may not hold. It is also possible that the Lyapunov-regularity of a DAE system does not imply the Lyapunov-regularity of its adjoint and vice versa.

Example 22 Consider the DAE system

$$
\begin{array}{rrr}
e^{a t} \dot{x}_{1} & = & e^{a t} \lambda x_{1}+x_{2} \\
0 & = & -e^{b t} x_{2}
\end{array}
$$

for $t \in \mathbb{I}$, with constants $a \leq 0, b \leq 0$ and $\lambda \in \mathbb{R}$. The adjoint system is

$$
\begin{aligned}
e^{a t} \dot{y}_{1} & =-\left(e^{a t} \lambda+a e^{a t}\right) y_{1} \\
0 & =-y_{1}+e^{b t} y_{2} .
\end{aligned}
$$

It is easy to see that both (23) and (24) are Lyapunov-regular. The only Lyapunov exponent for (23) is $\lambda$, while the only Lyapunov exponent for (24) is $-\lambda-a-b$. So, the Perron identity (20) between the Lyapunov exponents does not hold if $a+b \neq 0$. In addition, the Lyapunov exponent of (24) is not necessarily equal to that of its underlying ODE. Note that in this example all the coefficient matrices are bounded.

Example 23 Consider the systems (23) and (24) as in Example 22 but assume that $a$ is positive, i. e. the leading coefficient matrix is unbounded and assume that $\lambda$ is given by the time-varying function $\lambda(t)=\sin (\ln (t+1))+\cos (\ln (t+1))$. Then, the Lyapunov spectrum of $(23)$ is $[-1,1]$ and that of the adjoint $(24)$ is $[-1-a-b, 1-a-b]$. Neither the DAEs nor their underlying ODEs are Lyapunov-regular. However, if $a+b=2$, then (20) holds for the upper Lyapunov exponents but the spectra of the DAE and its adjoint are not symmetric at all.

As we have defined it, Lyapunov-regularity is an asymptotic property of solutions to a DAE system. Hence, the Lyapunov-regularity definition presented here seems to be more natural than that based on the Perron identity (20) given in [18]. Clearly, if the conditions (17) and (19) hold, then the different definitions of Lyapunov-regularity are equivalent.

The following two examples demonstrate the effect of the algebraic constraint on the dynamical behavior of solutions. We stress that again in these examples the coefficient matrices are bounded.

Example 24 Consider the DAE system

$$
\begin{aligned}
\dot{x}_{1} & =-x_{1}, \\
0 & =x_{1}-e^{-t+t \sin (t)} x_{2} .
\end{aligned}
$$

Here the underlying ODE is Lyapunov-regular, but the DAE itself is not. Conversely, consider the DAE

$$
\begin{aligned}
\dot{x}_{1} & =\left[\sin (\ln (t+1)+\cos (\ln (t+1))] x_{1},\right. \\
0 & =-x_{1}+e^{t \sin (\ln (t+1))-t} x_{2} .
\end{aligned}
$$

Here the DAE is Lyapunov-regular but the underlying ODE is not.

Example 25 The DAE system

$$
\begin{aligned}
\dot{x}_{1} & =-3 x_{1}+e^{t \sin (t)-t} x_{2}, \\
0 & =e^{-t} x_{2}
\end{aligned}
$$


is Lyapunov-regular. However, its adjoint system

$$
\begin{aligned}
\dot{y}_{1} & =3 y_{1}, \\
0 & =-e^{t \sin (t)-t} y_{1}-e^{-t} y_{2},
\end{aligned}
$$

is not.

Remark 26 We see from these examples that not the boundedness of the original coefficient matrices, but rather the boundedness of the terms in (17), (19) is relevant. For example, multiplying the algebraic equation in (9) from the left with a nonsingular matrix function of size $a \times a$ may change the boundedness of the coefficient matrix $A$. However, the validity of (17) is invariant under this transformation. Using the relation between the coefficients of (9) and those of (13), which follows by the proof of Theorem 12, one may easily reformulate the conditions (17), (19) in term of the original data, i.e., the coefficients of (9). Of course, the derivative of the matrix function $\hat{Q}$ appearing in Theorem 12 will be involved in the reformulated conditions. Furthermore, since $E_{1}=\left[\begin{array}{ll}\hat{E}_{11} & 0\end{array}\right] \hat{Q}$, the assumption on the growth rate of $\hat{E}_{11}$ in (19) will automatically imply the same on the growth rate of the original coefficient $E$.

In this section we have introduced the concepts of Lyapunov spectra and Lyapunov-regularity for strangeness-free DAEs of the form (9). Since these concepts only depend on the solution of the DAE and not on the representation of the system of DAEs, whether it is in the form (9) or in the general form (1), we immediately have all the results also for DAEs in the general form.

\subsection{Stability of Lyapunov exponents}

The analysis performed in the last subsection changes substantially if the DAE (9) is subject to perturbations, i. e. if one studies perturbed DAEs

$$
(E(t)+\Delta E(t)) \dot{x}=(A(t)+\Delta A(t)) x, \quad t \in \mathbb{I},
$$

with perturbation functions $\Delta E(t), \Delta A(t)$. If we allow general perturbations, then it is very difficult to analyze the behavior of the system due to the fact that the strangeness-index may change or the solvability of the system may be destroyed, see [10,34,63]. The complete perturbation analysis for this case is still an open problem even for constant coefficient systems. For this reason we require that the pair of perturbation functions $(\Delta E, \Delta A), \Delta E, \Delta A \in C\left(\mathbb{I}, \mathbb{R}^{n \times n}\right)$ are sufficiently smooth such that by applying a similar orthogonal transformation as from (9) to (13) (but not the same), we obtain

$$
\left[\begin{array}{cc}
\hat{E}_{11}+\Delta \hat{E}_{11} & 0 \\
0 & 0
\end{array}\right] \dot{\hat{x}}=\left[\begin{array}{ll}
\hat{A}_{11}+\Delta \hat{A}_{11} & \hat{A}_{12}+\Delta \hat{A}_{12} \\
\hat{A}_{21}+\Delta \hat{A}_{21} & \hat{A}_{22}+\Delta \hat{A}_{22}
\end{array}\right] \hat{x}, \quad t \in \mathbb{I} .
$$

If this is the case then we say that the perturbations are admissible.

Lemma 27 Consider a strangeness-free DAE of the form (9) and the set $\mathcal{P}$ of all pairs of admissible perturbation functions $(\Delta E, \Delta A)$ such that in the transformed systems (26) the blocks $\hat{E}_{11}$ and $\hat{A}_{22}$ are still invertible and have bounded inverses. If $(\Delta E, \Delta A) \in \mathcal{P}$ is sufficiently small, then (26) remains strangeness-free.

Proof. The assertion follows, since for sufficiently small admissible pairs of perturbations $(\Delta E, \Delta A)$ the functions $I+\hat{E}_{11}^{-1} \Delta \hat{E}_{11}$ and $I+\hat{A}_{22}^{-1} \Delta \hat{A}_{22}$ remain nonsingular.

If the unperturbed DAE systems corresponding to the transformed system (26) has boundedly invertible blocks $\hat{E}_{11}$ and $\hat{A}_{22}$, then we call these DAEs robustly strangeness-free. In the following we restrict ourselves to robustly strangeness-free DAE systems under admissible perturbations. 
Definition 28 The upper Lyapunov exponents $\lambda_{1}^{u} \geq \ldots \geq \lambda_{d}^{u}$ of (13) are said to be stable if for any $\epsilon>0$, there exists $\delta>0$ such that the conditions $\sup _{t}\|\Delta E(t)\|<\delta$, $\sup _{t}\|\Delta A(t)\|<\delta$ on the perturbations imply that the perturbed DAE system (26) is strangeness-free and

$$
\left|\lambda_{i}^{u}-\gamma_{i}^{u}\right|<\epsilon, \quad \text { for all } i=1,2, \ldots, d,
$$

where the $\gamma_{i}^{u}$ are the ordered upper Lyapunov exponents of the perturbed system (26).

The DAE system (13) and the perturbed system (26) are called asymptotically equivalent if they are strangeness-free and

$$
\lim _{t \rightarrow \infty}\|\Delta E(t)\|=\lim _{t \rightarrow \infty}\|\Delta A(t)\|=0 .
$$

It is clear that the stability of upper Lyapunov exponents and the asymptotic equivalence of DAE systems are invariant under strong global kinematic equivalence transformations. Since the Lyapunov exponents do not depend on the behavior of the coefficient matrices on a finite interval, we have the following result (see also [1, Theorem 5.2.1] or [27, Theorem 3.1]).

Theorem 29 Suppose that the DAE system (13) and the perturbed system (26) are asymptotically equivalent. Then the stability of the Lyapunov exponents of (13) implies $\lambda_{i}^{u}=\gamma_{i}^{u}$, for all $i=$ $1,2, \ldots, d$, where again the $\gamma_{i}^{u}$ are the ordered upper Lyapunov exponents of the perturbed system (26).

Proof. Due to the asymptotic equivalence of the two systems, given an arbitrary $\epsilon>0$, there exists $T>0$ (sufficiently large) such that

$$
\sup _{t \geq T}\|\Delta E(t)\|<\delta, \quad \sup _{t \geq T}\|\Delta A(t)\|<\delta
$$

where $\delta$ (depending on $\epsilon$ ) is as in Definition 28. By definition, the Lyapunov exponents are invariant with respect to changes occurring in the coefficient matrices on a finite interval $[0, T]$. On the other hand, due to the stability (of the Lyapunov exponents), the inequalities (27) imply that

$$
\left|\lambda_{i}^{u}-\gamma_{i}^{u}\right|<\epsilon, \quad \text { for all } i=1,2, \ldots, d .
$$

Since $\epsilon$ can be chosen arbitrarily small, the proof is complete.

As our next step we extend the concept of integral separation to DAEs.

Definition 30 A minimal fundamental solution matrix X for (9) (or (13)) is called integrally separated if for $i=1,2, \ldots, d-1$ there exist constants $\beta>0$ and $\gamma>0$ such that

$$
\frac{\left\|X(t) e_{i}\right\|}{\left\|X(s) e_{i}\right\|} \cdot \frac{\left\|X(s) e_{i+1}\right\|}{\left\|X(t) e_{i+1}\right\|} \geq \gamma e^{\beta(t-s)},
$$

for all $t, s$ with $t \geq s \geq 0$. If a DAE system has an integrally separated minimal fundamental solution matrix, then we say it has the integral separation property.

Analogous to the result for ODEs, see e.g. [26], we then have the following facts.

Proposition 31 Consider a strangeness-free DAE system of the form (13).

1. If (13) is integrally separated then the same holds for any globally kinematically equivalent system, i. e. also for (9).

2. If (13) is integrally separated, then it has pairwise distinct upper and pairwise distinct lower Lyapunov exponents.

3. Suppose that $\hat{A}_{22}^{-1} \hat{A}_{21}$ is bounded. Then, the DAE system (13) is integrally separated if and only if and the underlying $O D E(15)$ is integrally separated. 
Proof.

1. Let $\hat{X}$ be a fundamental solution matrix of (13). Suppose that, under a global kinematic equivalence transformation, the transformed fundamental solution matrix $\tilde{X}$ is given as $\tilde{X}=V \hat{X}$, where $V$ is smooth, and $V$ as well as $V^{-1}$ are bounded. Then,

$$
\frac{\left\|V(t) \hat{X}(t) e_{i}\right\|}{\left\|V(s) \hat{X}(s) e_{i}\right\|} \geq \frac{1}{\operatorname{cond}(V)} \frac{\left\|\hat{X}(t) e_{i}\right\|}{\left\|\hat{X}(s) e_{i}\right\|}
$$

for all $t, s$ and $i=1,2, \ldots, d$, where

$$
\operatorname{cond}(V):=\sup _{t, s}\|V(t)\|\left\|V^{-1}(s)\right\| .
$$

A similar estimate for $i+1$ with the change of variables $t \leftrightarrow s$, yields

$$
\frac{\left\|V(s) \hat{X}(s) e_{i+1}\right\|}{\left\|V(t) \hat{X}(t) e_{i+1}\right\|} \geq \frac{1}{\operatorname{cond}(V)} \frac{\left\|\hat{X}(s) e_{i+1}\right\|}{\left\|\hat{X}(t) e_{i+1}\right\|} .
$$

Multiplying the left and right side of these two inequalities for $i$ and $i+1$, we obtain

$$
\frac{\left\|V(t) \hat{X}(t) e_{i}\right\|}{\left\|V(s) \hat{X}(s) e_{i}\right\|} \cdot \frac{\left\|V(s) \hat{X}(s) e_{i+1}\right\|}{\left\|V(t) \hat{X}(t) e_{i+1}\right\|} \geq \frac{1}{[\operatorname{cond}(V)]^{2}} \frac{\left\|\hat{X}(t) e_{i}\right\|}{\left\|\hat{X}(s) e_{i}\right\|} \cdot \frac{\left\|\hat{X}(s) e_{i+1}\right\|}{\left\|\hat{X}(t) e_{i+1}\right\|} \geq \frac{1}{[\operatorname{cond}(V)]^{2}} \gamma e^{\beta(t-s)},
$$

for all $t, s$ and $i=1,2, \ldots, d-1$, which immediately yields the assertion.

2. This part is immediate.

3. The proof is similar to that of Part 1. We use again the estimates (18) between the columns of $\hat{X}$ and the corresponding columns of the fundamental solution for (15).

Remark 32 Note that the integral separation property is of a rather uniform nature. It is stronger than the property that

$$
\frac{\left\|X(t) e_{i}\right\|}{\left\|X(t) e_{i+1}\right\|} \geq \gamma e^{\beta t}, \quad t \in \mathbb{I},
$$

which is sufficient for the Lyapunov exponents to be pairwise distinct. This is the reason, why in Part 3. of Proposition 31 we require a stronger assumption than the condition (17) that we have used before.

Theorem 33 Suppose that the coefficient matrices in (13) are such that

$$
\hat{A}_{22}^{-1} \hat{A}_{21}, \hat{A}_{12} \hat{A}_{22}^{-1}, \hat{E}_{11}, \hat{E}_{11}^{-1}\left(\hat{A}_{11}-\hat{A}_{12} \hat{A}_{22}^{-1} \hat{A}_{21}\right) \text { are bounded. }
$$

If the system (13) has d pairwise distinct upper and pairwise distinct lower Lyapunov exponents and they are stable, then the system admits integral separation. Conversely, if there exists an integrally separated fundamental solution matrix to (13), then the system has $d$ stable pairwise distinct upper and stable pairwise distinct lower Lyapunov exponents.

Proof. Under the boundedness assumption of $\hat{A}_{22}^{-1} \hat{A}_{21}$, the DAE system (13) possesses the same Lyapunov exponents as its underlying ODE (15). The boundedness conditions (28) imply that if the perturbations $\Delta E$ and $\Delta A$ are small enough, then the underlying explicit ODE

$$
\dot{\hat{x}}_{1}=\hat{A} \hat{x}_{1}=\hat{E}_{11}^{-1}\left(\hat{A}_{11}-\hat{A}_{12} \hat{A}_{22}^{-1} \hat{A}_{21}\right) \hat{x}_{1}
$$

is only affected by a small perturbation in the coefficient matrix $\hat{A}$. By invoking Part 3 of Proposition 31 and the well-known result for ODEs [1], see also [26], the proof for system (13) follows. $\square$ 
Remark 34 Theorem 31 is stated for both the upper and the lower Lyapunov exponents. But one should note that although both upper and lower Lyapunov exponents are pairwise distinct, the Lyapunov spectral intervals may intersect each other, see Example 40 below.

Unlike the case of ODEs, the integral separation of a DAE system does not automatically imply that of its adjoint system.

Theorem 35 Consider a strangeness-free DAE system of the form (13) and suppose that $\hat{A}_{22}^{-1} \hat{A}_{21}$, $\hat{A}_{12} \hat{A}_{22}^{-1}, \hat{E}_{11}$, and $\hat{E}_{11}^{-1}$ are bounded. Then, the system has an integrally separated fundamental solution matrix if and only if its adjoint (12) has an integrally separated fundamental solution matrix.

Proof. The proof follows immediately by using the structure of the fundamental solution matrices of (13), the Lagrange identity, the first statement of Theorem 19, and Part 3. of Proposition 31. $\square$

Recall that if the columns of $\hat{X}_{1}$ form a normal basis for (15) then the columns of $Y_{1}:=$ $\hat{E}_{11}^{-T} \hat{X}_{1}^{-T}$ form a normal basis for the adjoint. But, as the following example shows, without the boundedness conditions in Theorem 35, the integral separation of the DAE (13) does not imply the integral separation of the underlying ODE (15).

Example 36 Consider the DAE system

$$
\begin{aligned}
\dot{x}_{1} & =x_{1}+x_{2}, \\
\dot{x}_{2} & =x_{2}, \\
0 & =x_{1}-x_{3}, \\
0 & =x_{2}-e^{-t} x_{4} .
\end{aligned}
$$

Clearly, the underlying ODE

$$
\left[\begin{array}{l}
\dot{x}_{1} \\
\dot{x}_{2}
\end{array}\right]=\left[\begin{array}{ll}
1 & 1 \\
0 & 1
\end{array}\right]\left[\begin{array}{l}
x_{1} \\
x_{2}
\end{array}\right]
$$

is not integrally separated, but the DAE system has a minimal fundamental solution

$$
X(t)=\left[\begin{array}{cc}
e^{t} & t e^{t} \\
0 & e^{t} \\
e^{t} & t e^{t} \\
0 & e^{2 t}
\end{array}\right],
$$

which is integrally separated. This illustrates the need for the boundedness assumptions in (28).

\subsection{Bohl exponents and Sacker-Sell spectrum}

Another concept that can be used to describe the behavior of solutions to ordinary differential equations is that of Bohl exponents [7], see also [20]. The extension of this concept to DAEs is straightforward.

Definition 37 Let $x$ be a nontrivial solution of (9). The (upper) Bohl exponent $\kappa_{B}^{u}(x)$ of this solution is the greatest lower bound of all those values $\rho$ for which there exist constants $N_{\rho}>0$ such that

$$
\|x(t)\| \leq N_{\rho} e^{\rho(t-s)}\|x(s)\|
$$

for any $t \geq s \geq 0$. If such numbers $\rho$ do not exist, then one sets $\kappa_{B}^{u}(x)=+\infty$.

Similarly, the lower Bohl exponent $\kappa_{B}^{\ell}(x)$ is the least upper bound of all those values $\rho^{\prime}$ for which there exist constants $N_{\rho}^{\prime}>0$ such that

$$
\|x(t)\| \geq N_{\rho}^{\prime} e^{\rho^{\prime}(t-s)}\|x(s)\|, \quad 0 \leq s \leq t .
$$

The interval $\left[\kappa_{B}^{\ell}(x), \kappa_{B}^{u}(x)\right]$ is called the Bohl interval of the solution $x$. 
It follows directly from the definition, that Lyapunov exponents and Bohl exponents are related via

$$
\kappa_{B}^{\ell}(x) \leq \lambda^{\ell}(x) \leq \lambda^{u}(x) \leq \kappa_{B}^{u}(x) .
$$

Bohl exponents characterize the uniform growth rate of solutions, while Lyapunov exponents simply characterize the growth rate of solutions departing from $t=0$.

Remark 38 The Bohl exponent of linear ODEs, which was introduced first in [7], has been proven to be a useful tool in the qualitative theory and in the control of finite as well as infinite dimensional linear systems. Numerous properties of Bohl exponent are discussed in [20]. Though less wellknown than the famous characteristic number introduced by Lyapunov, the Bohl exponent is often preferable, since it is stable with respect to small perturbations occurring in the coefficient matrix. For this reason, the Bohl exponent was used for characterizing the robust stability of linear systems, see e. g., $[44,16]$ and the references therein.

It is straightforward to extend the formulas for Bohl exponents of ODEs, see e.g. [20], to DAEs, i.e.

$$
\kappa_{B}^{u}(x)=\limsup _{s, t-s \rightarrow \infty} \frac{\ln \|x(t)\|-\ln \|x(s)\|}{t-s}, \quad \kappa_{B}^{\ell}(x)=\liminf _{s, t-s \rightarrow \infty} \frac{\ln \|x(t)\|-\ln \|x(s)\|}{t-s} .
$$

It is also well-known for linear ODEs $\dot{x}=A(t) x$ that if the coefficient matrix function $A(t)$ is integrally bounded, i.e., if

$$
\sup _{t \geq 0} \int_{t}^{t+1}\|A(s)\| d s<\infty
$$

then the Bohl exponents are finite, see [20]. For a continuous bounded function $A$, this condition trivially holds. In summary we have the following properties of Bohl exponents for DAEs.

Proposition 39 Consider the DAE system (9) and the transformed system (13). Then we have the following properties of Bohl exponents.

1. Bohl exponents are invariant under global kinematical equivalence transformations.

2. Consider a minimal fundamental solution matrix $\hat{X}$ for (13). If $\hat{A}_{22}^{-1} \hat{A}_{21}$ is bounded, then the Bohl intervals for the columns of $\hat{X}$ are exactly the Bohl intervals for the corresponding fundamental solution matrix $\hat{X}_{1}$ of the underlying $O D E(15)$.

3. If the Bohl intervals of the columns of a minimal fundamental solution matrix $X$ of (9) are $d$ disjoint closed intervals, then $X$ has integrally separated columns.

4. If the columns of a fundamental solution matrix $X$ of (9) are integrally separated, then the upper (or the lower) Bohl exponents of the columns of $X$ columns are distinct, but the Bohl intervals may intersect each other.

Proof.

1. Suppose that $V \in C^{1}\left(\mathbb{I}, \mathbb{R}^{n \times n}\right)$ such that $V$ and $V^{-1}$ are bounded. Let $x$ be an arbitrary solution to (9). Since

$$
\frac{\|V(t) x(t)\|}{\|V(s) x(s)\|} \geq \frac{1}{\operatorname{cond}(V)} \frac{\|x(t)\|}{\|x(s)\|}
$$

we have

$$
\frac{\ln \|V(t) x(t)\|-\ln \|V(s) x(s)\|}{t-s} \geq \frac{\ln \|x(t)\|-\ln \|x(s)\|-\ln \operatorname{cond}(V)}{t-s} .
$$

Taking the limsup on both sides as $s, t-s$ tend to $\infty$, we obtain

$$
\kappa_{B}^{u}(V x) \geq \kappa_{B}^{u}(x)
$$


Conversely, we have $x=V^{-1} V x$, hence similarly we obtain

$$
\kappa_{B}^{u}(x) \geq \kappa_{B}^{u}(V x)
$$

As a consequence, we have $\kappa_{B}^{u}(V x)=\kappa_{B}^{u}(x)$. The proof for the lower Bohl exponents is obtained analogously.

2. The proof is similar to that of Theorem 14 .

3. Without loss of generality, we assume that the Bohl intervals of $X$ are ordered decreasingly. For the sake of simplicity, it suffices to consider the first two columns $x_{1}$ and $x_{2}$. By the definition of the Bohl exponents, there exist positive constants $N_{i}, M_{i}, i=1,2$ such that

$$
N_{i} e^{\kappa_{i}^{l}(t-s)}\left\|x_{i}(s)\right\| \leq\left\|x_{i}(t)\right\| \leq M_{i} e^{\kappa_{i}^{u}(t-s)}\left\|x_{i}(s)\right\|, \quad \text { for all } t \geq s,
$$

for $i=1,2$, where $\kappa_{i}^{l}$ and $\kappa_{i}^{u}$ are lower and upper Bohl exponents for $x_{i}$, respectively. Then, it is easy to see that

$$
\frac{\left\|x_{1}(t)\right\|}{\left\|x_{1}(s)\right\|} \frac{\left\|x_{2}(s)\right\|}{\left\|x_{2}(t)\right\|} \geq \frac{N_{1} e^{\kappa_{1}^{l}(t-s)}}{M_{2} e^{\kappa_{2}^{u}(t-s)}}=\frac{N_{1}}{M_{2}} e^{\left(\kappa_{1}^{l}-\kappa_{2}^{u}\right)(t-s)}
$$

holds for all $t \geq s$. Since the intervals $\left[\kappa_{1}^{l}, \kappa_{1}^{u}\right]$ and $\left[\kappa_{2}^{l}, \kappa_{2}^{u}\right]$ are disjoint, the positivity of $\left(\kappa_{1}^{l}-\kappa_{2}^{u}\right)$ is obvious, which verifies the integral separation of $x_{1}$ and $x_{2}$.

4. For the converse statement, we consider again the first two columns $x_{1}$ and $x_{2}$. By the integral separation property, there exist positive constants $\beta, \gamma$ such that

$$
\frac{\left\|x_{1}(t)\right\|}{\left\|x_{1}(s)\right\|} \frac{\left\|x_{2}(s)\right\|}{\left\|x_{2}(t)\right\|} \geq \gamma e^{\beta(t-s)} \quad t \geq s,
$$

or equivalently

$$
\frac{\left\|x_{1}(t)\right\|}{\left\|x_{1}(s)\right\|} \geq \gamma e^{\beta(t-s)} \frac{\left\|x_{2}(t)\right\|}{\left\|x_{2}(s)\right\|}
$$

which implies

$$
\frac{\ln \left\|x_{1}(t)\right\|-\ln \left\|x_{1}(s)\right\|}{t-s} \geq \frac{\ln \gamma+\beta(t-s)+\ln \left\|x_{2}(t)\right\|-\ln \left\|x_{2}(s)\right\|}{t-s} .
$$

Taking the limsup on both sides of the above inequality as $s, t-s$ tend to infinity, we obtain

$$
\limsup _{s, t-s \rightarrow \infty} \frac{\ln \left\|x_{1}(t)\right\|-\ln \left\|x_{1}(s)\right\|}{t-s} \geq \limsup _{s, t-s \rightarrow \infty} \frac{\ln \left\|x_{1}(t)\right\|-\ln \left\|x_{1}(s)\right\|}{t-s},
$$

which implies that $\kappa_{1}^{u}>\kappa_{2}^{u}$. By taking the liminf instead of the limsup, we obtain the result for the lower Bohl exponents.

口

Even though we have shown that integral separation implies distinct upper and lower Bohl exponents, the Bohl intervals may still overlap as the following example demonstrates.

Example 40 Consider the system

$$
\begin{aligned}
& \dot{x}_{1}(t)=[\sin (\ln t)+\cos (\ln t)] x_{1}(t), \\
& \dot{x}_{2}(t)=[\sin (\ln t)+\cos (\ln t)-1] x_{2}(t),
\end{aligned}
$$

with $t \geq 1$ that is an extension to DAEs of an example by Perron [65]. It is easy to see that this system is integrally separated. However, the Bohl intervals $[-\sqrt{2}, \sqrt{2}]$ and $[-\sqrt{2}-1, \sqrt{2}-1]$ are clearly not disjoint. Similarly, also the Lyapunov spectral intervals of this system $[-1,1]$ and $[-2,0]$ overlap. 
In order to extend the concept of exponential dichotomy to DAEs, we first introduce shifted DAE systems.

Definition 41 Consider a strangeness-free DAE of the form (9). For $\lambda \in \mathbb{R}$, the DAE system

$$
E(t) \dot{x}=[A(t)-\lambda E(t)] x, \quad t \in \mathbb{I},
$$

is called a shifted DAE system.

By the transformation of Theorem 12, the shifted DAE transforms as

$$
\left[\begin{array}{cc}
\hat{E}_{11} & 0 \\
0 & 0
\end{array}\right] \dot{\hat{x}}=\left[\begin{array}{cc}
\hat{A}_{11}-\lambda \hat{E}_{11} & \hat{A}_{12} \\
\hat{A}_{21} & \hat{A}_{22}
\end{array}\right] \hat{x}, \quad t \in \mathbb{I},
$$

and clearly, the shifted DAE system inherits the strangeness-free property from the original DAE.

In the previous subsection, we have seen that minimal fundamental solution matrices are useful in the analysis of Lyapunov exponents. Unfortunately, they do not have the semi-group property as fundamental solutions in the ODE case have. However, for strangeness-free systems it is easy to introduce a (unique) maximal fundamental solution matrix $X$ which possesses a semi-group property by demanding that it satisfies the projected initial condition

$$
E\left(t_{0}\right)\left(X\left(t_{0}\right)-I_{n}\right)=0 .
$$

Example 42 Condition (33) is, however, not the right condition, if the system is not strangenessfree. Consider

$$
E(t) \dot{x}(t)=A(t) x(t), \quad t \in \mathbb{I},
$$

with

$$
E(t)=\left[\begin{array}{cc}
0 & 0 \\
1 & -t
\end{array}\right], \quad A(t)=\left[\begin{array}{cc}
-1 & t \\
0 & 0
\end{array}\right]
$$

see $[46,50]$. The strangeness index of this system is 1 . It is easy to see that the trivial solution $X \equiv 0$ is the only maximal fundamental matrix solution, but it does not satisfy (33).

In the following, when it is necessary to emphasize the dependence of the fundamental solution matrix on the initial time $t_{0}$, we write $X\left(t, t_{0}\right)$.

In the case of the transformed strangeness-free system (13), a maximal fundamental solution matrix that satisfies the corresponding projected initial condition (33) is easily obtained as

$$
\hat{X}\left(t, t_{0}\right)=\left[\begin{array}{cc}
\hat{X}_{1}\left(t, t_{0}\right) & 0 \\
\hat{A}_{22}^{-1} \hat{A}_{21}(t) \hat{X}_{1}\left(t, t_{0}\right) & 0
\end{array}\right],
$$

where $\hat{X}_{1}$ is a fundamental solution matrix for (15) satisfying $\hat{X}_{1}\left(t_{0}, t_{0}\right)=I_{d}$. For this maximal fundamental solution matrix $\hat{X}\left(t, t_{0}\right)$, we introduce the generalized inverse matrix function $\hat{X}^{-}\left(t, t_{0}\right)$ defined by

$$
\hat{X}^{-}\left(t, t_{0}\right):=\left[\begin{array}{cc}
\hat{X}_{1}^{-1}\left(t, t_{0}\right) & 0 \\
\hat{A}_{22}^{-1} \hat{A}_{21}\left(t_{0}\right) \hat{X}_{1}^{-1}\left(t, t_{0}\right) & 0
\end{array}\right],
$$

for $t \geq t_{0}$. The matrix functions $\hat{X}$ and $\hat{X}^{-}$satisfy the following relations.

Proposition 43 Let $\hat{X}$ as in (34) be the maximal fundamental solution matrix of (13) and let $\hat{X}^{-}$be as in (35). Then for $t_{1} \geq t_{0}$, the following identities hold.

1. $\hat{X}\left(t_{1}, t_{0}\right) \hat{X}^{-}\left(t_{1}, t_{0}\right) \hat{X}\left(t_{1}, t_{0}\right)=\hat{X}\left(t_{1}, t_{0}\right)$,

2. $\hat{X}^{-}\left(t_{1}, t_{0}\right) \hat{X}\left(t_{1}, t_{0}\right) \hat{X}^{-}\left(t_{1}, t_{0}\right)=\hat{X}^{-}\left(t_{1}, t_{0}\right)$,

3. $\hat{X}\left(t_{1}, t_{0}\right) \hat{X}^{-}\left(t_{1}, t_{0}\right)=\left[\begin{array}{cc}I & 0 \\ \hat{A}_{22}^{-1}\left(t_{1}\right) \hat{A}_{21}\left(t_{1}\right) & 0\end{array}\right]$, 


$$
\text { 4. } \hat{X}^{-}\left(t_{1}, t_{0}\right) \hat{X}\left(t_{1}, t_{0}\right)=\left[\begin{array}{cc}
I & 0 \\
\hat{A}_{22}^{-1}\left(t_{0}\right) \hat{A}_{21}\left(t_{0}\right) & 0
\end{array}\right] .
$$

Vice versa, these four properties define the matrix $\hat{X}^{-}$uniquely.

Furthermore, $\hat{X}$ satisfies the semigroup property, i.e. for $t_{2} \geq t_{1} \geq t_{0}$, we have

$$
\hat{X}\left(t_{2}, t_{1}\right) \hat{X}\left(t_{1}, t_{0}\right)=\hat{X}\left(t_{2}, t_{0}\right) .
$$

Proof. By using the formulae (34) and (35), the identities 1.-4. as well as the semigroup property are easily verified by elementary matrix calculations.

The identities 1.-2. in Proposition 43 mean that $X^{-}\left(t_{1}, t_{0}\right)$ is a reflexive generalized inverse of $X\left(t_{1}, t_{0}\right)$, while the identities 3.-4. guarantee that this generalized inverse is unique, see $[5,6]$.

In the following, for ease of notation, we use $t_{0}=0$ and the abbreviation $\hat{X}(t):=\hat{X}(t, 0)$. We then introduce the concept of exponential dichotomy for DAEs as in [57].

Definition 44 The semi-implicit DAE system (13) is said to have an exponential dichotomy if for a maximal fundamental solution matrix $\hat{X}(t)$, there exists a projection matrix $P \in \mathbb{R}^{d \times d}$ and constants $\alpha, \beta>0$, and $K, L \geq 1$ such that

$$
\begin{gathered}
\left\|\hat{X}(t)\left[\begin{array}{cc}
P & 0 \\
0 & 0
\end{array}\right] \hat{X}^{-}(s)\right\| \leq K e^{-\alpha(t-s)}, \quad t \geq s, \\
\left\|\hat{X}(t)\left[\begin{array}{cc}
I_{d}-P & 0 \\
0 & 0
\end{array}\right] \hat{X}^{-}(s)\right\| \leq L e^{\beta(t-s)}, \quad t \leq s .
\end{gathered}
$$

Furthermore, we say that a general DAE system (9) has an exponential dichotomy if there exists a global kinematical equivalence transformation that reduces (9) to the semi-implicit form and the reduced system has an exponential dichotomy.

For a strangeness-free DAE in the form (13), exponential dichotomy can again be characterized via the underlying ODE.

Theorem 45 The DAE system (13) has an exponential dichotomy if and only if $\hat{A}_{22}^{-1} \hat{A}_{21}$ is bounded and the corresponding underlying $O D E(15)$ has an exponential dichotomy.

Proof. Suppose that (36) holds. Using the structure of $\hat{X}$ and $\hat{X}^{-}$, we can rewrite (36) as

$$
\begin{gathered}
\left\|\left[\begin{array}{c}
\hat{X}_{1}(t) P \hat{X}_{1}^{-1}(s) \\
\hat{A}_{22}^{-1}(t) \hat{A}_{21}(t) \hat{X}_{1}(t) P \hat{X}_{1}^{-1}(s)
\end{array}\right]\right\| \leq K e^{-\alpha(t-s)}, \quad t \geq s, \\
\hat{X}_{1}(t)\left(I_{d}-P\right) \hat{X}_{1}^{-1}(s) \\
\left\|\left[\begin{array}{c}
\hat{A}_{22}^{-1}(t) \hat{A}_{21}(t) \hat{X}_{1}(t)\left(I_{d}-P\right) \hat{X}_{1}^{-1}(s)
\end{array}\right]\right\| \leq L e^{\beta(t-s)}, \quad t \leq s,
\end{gathered}
$$

which implies that the underlying ODE (15) has an exponential dichotomy. Setting $t=s$, we obtain

$$
\left\|\left[\begin{array}{c}
I_{d} \\
\hat{A}_{22}^{-1}(t) \hat{A}_{21}(t)
\end{array}\right]\right\| \leq\left\|\hat{X}(t)\left[\begin{array}{ll}
P & 0 \\
0 & 0
\end{array}\right] \hat{X}^{-}(t)\right\|+\left\|\hat{X}(t)\left[\begin{array}{cc}
I_{d}-P & 0 \\
0 & 0
\end{array}\right] \hat{X}^{-}(t)\right\| \leq K+L, \quad t \in \mathbb{I},
$$

from which the boundedness of $\hat{A}_{22}^{-1}(t) \hat{A}_{21}(t)$ is clear.

If $\hat{A}_{22}^{-1} \hat{A}_{21}$ is bounded and (15) has an exponential dichotomy, then clearly the inequalities in (37) hold.

The following facts associated with exponential dichotomy of DAEs follow easily.

Proposition 46 Consider a strangeness-free DAE of the form (13) that has an exponential dichotomy. 
1. Every globally kinematically equivalent system has an exponential dichotomy, $i$. e. in particular the exponential dichotomic property is invariant under global kinematical equivalence transformations.

2. If a fundamental solution matrix $\hat{X}$ of (13) has an exponential dichotomy, then so does the fundamental solution matrix that fulfills the projected initial condition (33) at $t_{0}=0$. Furthermore, the projection $P$ can be chosen to be orthogonal.

Proof. The first part simply follows by the definition of exponential dichotomic DAE systems. By Theorem 45, to verify the second statement, it suffices to consider the underlying ODE system (15) and analyze its exponential dichotomy. Invoking [27, Lemma 6.1], the underlying ODE system (15) also admits an exponential dichotomy for its (principal) matrix solution that satisfies $\hat{X}_{1}(0)=I_{d}$ and the projection $P$ can be chosen to be orthogonal. Finally, note that the fundamental matrix solution for (13) constructed with this $\hat{X}_{1}$, see (34), is exactly the unique fundamental solution matrix that fulfills the projected initial condition.

After these preparations we can define Sacker-Sell spectra for DAEs.

Definition 47 The Sacker-Sell (or exponential dichotomy) spectrum of the DAE system (13) is defined by

$$
\Sigma_{S}:=\{\lambda \in \mathbb{R}, \text { the shifted DAE (32) does not have an exponential dichotomy }\} .
$$

The complement of $\Sigma_{S}$ is called the resolvent set for the DAE system (13).

The Sacker-Sell spectrum of the DAE system (9) is defined as the Sacker-Sell spectrum of its transformed DAE system (13).

With these definitions we have the following properties of Sacker-Sell spectra for DAEs.

Lemma 48 Consider the DAE system (13) and suppose that $\hat{A}_{22}^{-1} \hat{A}_{21}$ is bounded. Then,

1. the Sacker-Sell spectrum of the DAE system (13) is exactly the Sacker-Sell spectrum of the underlying $O D E$ (15). It consists of at most d closed intervals.

2. the Sacker-Sell spectrum of the DAE system (9) does not depend on the choice of an orthogonal change of basis that transforms it into the form (13).

Proof.

1. Consider an arbitrary $\lambda \in \mathbb{R}$. By Theorem 45, the shifted DAE system (32) with this $\lambda$ has an exponential dichotomy if and only if the corresponding shifted underlying ODE system has an exponential dichotomy. This implies that the resolvent set of (13) and that of (15) are exactly the same which proves the assertion. Since the dimension (the size) of the underlying ODE system (15) is $d$, it has been shown in [70] that the Sacker-Sell spectrum of (15) consists of at most $d$ closed intervals.

2. As a consequence of Proposition 46, Part 1, two globally kinematically equivalent semiimplicit DAE systems must possess the same Sacker-Sell spectrum. Therefore, the SackerSell spectrum of the DAE system (9) does not depend on the choice of a global kinematic equivalence transformation that transforms it into the form (13).

We also obtain the relationship of Sacker-Sell spectra and the integral separation property.

Theorem 49 Suppose that the Sacker-Sell spectrum of (9) is given by d disjoint closed intervals. Then there exists a minimal fundamental solution matrix of (9) with integrally separated columns. 
Proof. Without loss of generality, it is sufficient to consider the transformed system (13). By Theorem 45, the assumption implies that $\hat{A}_{22}^{-1} \hat{A}_{21}$ is bounded. Then, by Lemma 48, the SackerSell spectrum of the underlying ODE (15) consists of the same $d$ disjoint intervals. By invoking [27, Theorem 6.3], then (15) has an integrally separated fundamental solution matrix denoted by $\hat{X}_{1}$. Hence, by construction (see Part 3. of Proposition 31), there exists a corresponding fundamental solution for the DAE system (13) whose columns are integrally separated.

For the converse of this result we again need a boundedness condition.

Theorem 50 Suppose that for the DAE system (13), $\hat{A}_{22}^{-1} \hat{A}_{21}$ is bounded and there exists a minimal and integrally separated fundamental solution matrix $\hat{X}$. Then the Sacker-Sell spectrum for (13) is given exactly by the d (not necessarily disjoint) Bohl intervals associated with the columns of $\hat{X}$.

Proof. Because of the relations between (13) and its underlying ODE (15), the verification of the statement reduces to that for the underlying ODE system (15). Due to a theorem of Bylov (see [1, Corollary 5.3.2] or [26, Theorem 2.31]), there exists a kinematic equivalence transformation that transforms (15) into diagonal form. The diagonalized system obtained in this way is integrally separated as well and the Sacker-Sell spectrum for the diagonal ODE system is exactly the set of Bohl intervals for all scalar equations corresponding to the diagonal elements, see also [58, Lemma 21]. Since Bohl intervals are invariant under global kinematic equivalence transformations, the proof is complete.

Remark 51 As is well-known already for ODEs [58], if we take an arbitrary minimal fundamental solution matrix of (13), then the set of Bohl intervals associated with its columns is only a subset of the Sacker-Sell spectrum of (13). The integral separation assumption then ensures that the two sets coincide. We underline that the relation between the Bohl exponents and the Sacker-Sell spectrum of a scalar ODE [58, Lemma 21] has resulted in a more elegant interpretation of SackerSell intervals compared with the approach based on two associated planar systems in [26, 27].

Corollary 52 Consider the strangeness-free DAE system (9). Then the Lyapunov spectrum is contained in the Sacker-Sell spectrum, i.e. we have $\Sigma_{L} \subseteq \Sigma_{S}$.

Proof. Suppose that the columns of a fundamental solution matrix $X$ of (9) form a normal basis. Then, by definition, the Lyapunov spectrum is exactly the set of Lyapunov intervals for the columns of $X$. Since for an arbitrary solution $x$ of (9), the Lyapunov interval is contained in the Bohl interval and since the Bohl intervals are contained in the Sacker-Sell spectrum (see Remark 51), the proof is complete.

The following well-known example example of Perron [65] shows that Lyapunov spectral intervals can be strict subsets of Sacker-Sell spectral intervals.

Example 53 Consider the ODE

$$
\dot{x}=[\sin (\ln (t+1))+\cos (\ln (t+1))] x,
$$

with solution $x(t)=x(0) e^{(t+1) \sin (\ln (t+1))}$. The Lyapunov spectrum is given by $[-1,1]$, while the Bohl interval (and the Sacker-Sell spectrum) is given by $[-\sqrt{2}, \sqrt{2}]$, see [20] and [26].

Finally we analyze the relationship between the Sacker-Sell spectra of the DAE system (13) and its adjoint (21).

Lemma 54 Consider the implicit scalar differential equation

$$
e(t) \dot{x}=a(t) x, \quad t \in \mathbb{I},
$$


where $e, e^{-1}$, and $e^{-1}$ a are continuous and bounded. Let the Sacker-Sell spectrum associated with this system be given by the interval $[\alpha, \beta]$. Then the Sacker-Sell spectrum of the adjoint equation

$$
e(t) \dot{y}=-(a(t)+\dot{e}(t)) y
$$

is given by $[-\beta,-\alpha]$.

Proof. Without loss of generality, we assume that $e(t)>0$ for all $t \in \mathbb{I}$. Let the Sacker-Sell spectrum of the adjoint equation be denoted by $[\bar{\alpha}, \bar{\beta}]$. Due to $[58$, Proposition 22$]$, we have

$$
\alpha=\liminf _{s, t-s \rightarrow \infty} \frac{1}{t-s} \int_{s}^{t} \frac{a(\tau)}{e(\tau)} d \tau
$$

and

$$
\bar{\beta}=\limsup _{s, t-s \rightarrow \infty} \frac{-1}{t-s} \int_{s}^{t}\left[\frac{a(\tau)}{e(\tau)}+\frac{\dot{e}(\tau)}{e(\tau)}\right] d \tau .
$$

By some elementary manipulations, we obtain

$$
\bar{\beta}=\limsup _{s, t-s \rightarrow \infty} \frac{-1}{t-s}\left[\int_{s}^{t} \frac{a(\tau)}{e(\tau)} d \tau+\ln e(t)-\ln e(s)\right] .
$$

Since $e$ and $e^{-1}$ are bounded, we get

$$
\lim _{s, t-s \rightarrow \infty} \frac{1}{t-s}(\ln e(t)-\ln e(s))=0
$$

and, therefore, $\bar{\beta}=-\alpha$. The proof that $\bar{\alpha}=-\beta$ follows analogously.

Before we can prove the symmetry property for the Sacker-Sell spectra of the DAE and its adjoint, we need the following lemma.

Lemma 55 Consider an implicit ODE of the form

$$
E(t) \dot{x}=A(t) x
$$

with $E(t)$ nonsingular. Suppose that both $E(t)$ and $A(t)$ are continuous and that $E^{-1} A$ is bounded. Then there exist orthogonal matrix functions $U \in C\left(\mathbb{I}, \mathbb{R}^{n, n}\right)$ and $V \in C^{1}\left(\mathbb{I}, \mathbb{R}^{n, n}\right)$ such that the transformed matrix functions

$$
\mathcal{E}=\left[e_{i j}\right]=U^{T} E V \quad \text { and } \quad \mathcal{A}=\left[a_{i j}\right]=U^{T} A V-U^{T} E \dot{V}
$$

are both in upper triangular form.

Proof. We give a constructive proof. We want to determine triangular matrix functions $\mathcal{E}$ and $\mathcal{A}$ with

$$
\mathcal{A}=U^{T} A V-U^{T} E \dot{V}=U^{T} A V-U^{T} E V V^{T} \dot{V}=U^{T} A V-\mathcal{E} V^{T} \dot{V}
$$

and

$$
\mathcal{E}^{-1} \mathcal{A}=\mathcal{E}^{-1} U^{T} A V-V^{T} \dot{V}
$$

being both triangular. Due to the orthogonality of $V$ we must have that $S(V)=\left[s_{i, j}\right]:=V^{T} \dot{V}$ is skew-symmetric. Hence, the strictly lower triangular part of $S(V)$ can be determined first by the corresponding part of $W=\left[w_{i j}\right]=\mathcal{E}^{-1} U^{T} A V$ and the remaining part then is determined by the skew-symmetry of $S(V)$, i.e.,

$$
s_{i j}=\left\{\begin{array}{cl}
w_{i j}, & i>j, \\
0, & i=j, \\
-w_{j i}, & i<j,
\end{array} \quad 1 \leq i, j \leq d .\right.
$$


The orthogonal matrix function $V$ then solves the differential equation

$$
\dot{V}=V S(V) \text {. }
$$

At $t=0$, we can obtain $V(0)$ by using a $Q R$-factorization of an initial value for the fundamental solution matrix $X(0)$ associated with (39) say, the identity matrix. Since $W=\mathcal{E}^{-1} U^{T} A V=$ $V^{T} E^{-1} A V$, the boundedness of $E^{-1} A$ implies the boundedness of $W$, thus the boundedness of $S(V)$, as well. Thus, $V$ can be determined as the (unique) solution to the initial value problem (41). With this, a continuous orthogonal matrix function $U$ (as well as a triangular matrix function $\mathcal{E})$ is then determined via

$$
E V=U \mathcal{E}
$$

and this then uniquely determines $\mathcal{A}$.

It should be noted that the computation of the triangularization in (42) can actually be implemented numerically by using smooth $Q R$-factorizations, see [21, Subsection 2.1].

Using Lemma 55, we can now prove the following theorem.

Theorem 56 Consider the DAE system (13) with continuous differentiable $\hat{E}_{11}$. Suppose that $\hat{A}_{22}^{-1} \hat{A}_{21}, \hat{A}_{12} \hat{A}_{22}^{-1}, \hat{E}_{11}$, and $\hat{E}_{11}^{-1}$ are bounded. Then, the Sacker-Sell spectrum of (13) and that of the adjoint system are symmetric with respect to the origin, $i$. e., if $\left[\alpha_{i}, \beta_{i}\right],(1 \leq i \leq d)$ is an arbitrary Sacker-Sell spectral interval for (13), then $\left[-\beta_{i},-\alpha_{i}\right]$ is a Sacker-Sell spectral interval for the adjoint system and vice versa.

Proof. By Lemma 48, it suffices to consider the Sacker-Sell spectrum associated with the underlying ODEs (15) and (22). Then, using similar arguments as in [23, Lemma 1.2], the proof is straightforward. Here we give an alternative proof. By Lemma 55, there exist orthogonal matrix functions $U_{1}, V_{1} \in C^{1}\left(\mathbb{I}, \mathbb{R}^{d, d}\right)$ such that (15) is equivalently transformed to

$$
\mathcal{E}_{1} \dot{z}_{1}=\mathcal{A}_{1} z_{1}
$$

where $\mathcal{E}_{1}=U_{1}^{T} \hat{E}_{11} V_{1}, \mathcal{A}_{1}=U_{1}^{T} \hat{A}_{s} V_{1}-U_{1}^{T} \hat{E}_{11} \dot{V}_{1}$ are both upper triangular. Similarly, the equivalence transformation associated with $V_{1}, U_{1}$ transforms the adjoint equation (22) to

$$
\mathcal{E}_{1}^{T} \dot{\zeta}_{1}=-\left(A_{1}^{T}+\dot{\mathcal{E}}_{1}\right) \zeta_{1},
$$

with lower triangular coefficients. It has been shown in [31] that the Sacker-Sell spectrum of ODE systems in triangular form is exactly the union of the Sacker-Sell spectra associated with each scalar equations corresponding to the diagonal elements. By invoking Lemma 54, the proof is complete.

In this section we have introduced the concepts of Sacker-Sell (exponential dichotomy) spectra for strangeness-free DAEs of the form (9). Again, since these concepts only depend on the solution of the DAE and not on the representation of the system of DAEs, whether it is in the form (9) or in the general form (1), we immediately have all the results also for DAEs in the general form.

\subsection{Stability of the Sacker-Sell spectrum}

We have seen that for Lyapunov spectra of ODEs we do not always have stability under small perturbations and this instability may clearly carry over to the DAE case. On the other hand we have stability of Bohl spectra for ODEs and DAEs. It remains to analyze the stability properties of Sacker-Sell spectra for DAE systems of the form (9). Again we have to restrict ourselves to admissible perturbations, i. e. perturbations of the form (26). We assume that the boundedness condition (28) holds and to make our perturbation bounds quantitative, we assume that there 
exist positive constants $M_{i}, 1 \leq i \leq 4, N_{j}, j=1,2$ and $\delta$ such that for $t \in \mathbb{I}$ the following bounds are given,

$$
\begin{array}{l||l|l||l}
\sup _{t} \\
\sup _{t}
\end{array} \hat{E}_{11}^{-1}\left(\hat{A}_{11}-\hat{A}_{12} \hat{A}_{22}^{-1} \hat{A}_{21}\right)\left\|\leq M_{1}, \quad \sup _{t}\right\| \hat{A}_{22}^{-1}\left\|\leq M_{2}, \quad \sup _{t}\right\| \hat{A}_{22}^{-1} \hat{A}_{21} \| \leq M_{3},
$$

To derive the perturbation bounds, we wlll need the following Lemma.

Lemma 57 Consider a strangeness-free DAE in the form (13) and a perturbed system of the form (26) with the bounds (43). If

$$
\delta<\min \left\{M_{2}^{-1}, N_{2}^{-1}\right\}
$$

then the perturbed system (26) remains strangeness-free and we have the estimates

$$
\left\|\left(\hat{A}_{22}+\Delta \hat{A}_{22}\right)^{-1}\right\| \leq \frac{M_{2}}{1-M_{2} \delta}, \quad\left\|\left(\hat{E}_{11}+\Delta \hat{E}_{11}\right)^{-1}\right\| \leq \frac{N_{2}}{1-N_{2} \delta}, \quad t \in \mathbb{I} .
$$

Proof. We have

$$
\hat{A}_{22}+\Delta \hat{A}_{22}=\hat{A}_{22}\left(I+\hat{A}_{22}^{-1} \Delta \hat{A}_{22}\right) .
$$

Since $\left\|\hat{A}_{22}^{-1} \Delta \hat{A}_{22}\right\| \leq \delta M_{2}<1$, we conclude the matrix $\left(I+\hat{A}_{22}^{-1} \Delta \hat{A}_{22}\right)$ is invertible for all $t \in \mathbb{I}$ and the estimate

$$
\left\|\left(I+\hat{A}_{22}^{-1} \Delta \hat{A}_{22}\right)^{-1}\right\| \leq \frac{1}{1-M_{2} \delta}
$$

holds. Hence, the matrix $\hat{A}_{22}+\Delta \hat{A}_{22}$ is invertible for all $t \in \mathbb{I}$ as well, and

$$
\left\|\left(\hat{A}_{22}+\Delta \hat{A}_{22}\right)^{-1}\right\| \leq \frac{M_{2}}{1-M_{2} \delta} .
$$

The claim for $\hat{E}_{11}+\Delta \hat{E}_{11}$ is proved similarly. Note that the invertibility of these matrices implies the strangeness-free property of the perturbed DAE system (26).

Considering now the perturbed system (26), it is not difficult to verify that (26) satisfies the boundedness condition (28). Using the estimates in Lemma 57, we immediately see that

$$
\left\|\left(\hat{A}_{22}+\Delta \hat{A}_{22}\right)^{-1}\left(\hat{A}_{21}+\Delta \hat{A}_{21}\right)\right\| \leq \frac{M_{3}+M_{2} \delta}{1-M_{2} \delta}
$$

and we obtain an estimate for the perturbation arising in the coefficient of the underlying ODE associated with the perturbed system (26). This estimate is given by

$$
\left\|\left(\hat{A}_{12}+\Delta \hat{A}_{12}\right)\left(\hat{A}_{22}+\Delta \hat{A}_{22}\right)^{-1}\left(\hat{A}_{21}+\Delta \hat{A}_{21}\right)-\hat{A}_{12} \hat{A}_{22}^{-1} \hat{A}_{21}\right\| \leq \frac{M_{3}+M_{2} \delta}{1-M_{2} \delta}\left(1+M_{4}\right) \delta=: M_{5}(\delta) \delta,
$$

where $M_{5}(\delta)=M_{3}\left(1+M_{4}\right)+O(\delta)$ for sufficiently small $\delta$.

If we reformulate the underlying unperturbed and perturbed underlying ODEs explicitely as

$$
\dot{\hat{x}}_{1}=\hat{E}_{11}^{-1}\left(\hat{A}_{11}-\hat{A}_{12} \hat{A}_{22}^{-1} \hat{A}_{21}\right) \hat{x}_{1},
$$

and

$$
\dot{\hat{x}_{1}}=\left(\bar{E}_{11}\right)^{-1}\left(\bar{A}_{11}-\bar{A}_{12} \bar{A}_{22}^{-1} \bar{A}_{21}\right) \bar{x}_{1},
$$

respectively, with $\bar{E}_{11}=\hat{E}_{11}+\Delta \hat{E}_{11}$ and $\bar{A}_{i j}=\hat{A}_{i j}+\Delta \hat{A}_{i j}, i, j=1,2$, then setting $\hat{A}_{s}=$ $\hat{A}_{11}-\hat{A}_{12} \hat{A}_{22}^{-1} \hat{A}_{21}$ and $\bar{A}_{s}=\bar{A}_{11}-\bar{A}_{12} \bar{A}_{22}^{-1} \bar{A}_{21}$, we obtain the perturbation bound

$\left\|\bar{E}_{11}^{-1} \bar{A}_{s}-\hat{E}_{11}^{-1} \hat{A}_{s}\right\|=\left\|\left(1+\hat{E}_{11}^{-1} \Delta \hat{E}_{11}\right)^{-1} \hat{E}_{11}^{-1} \bar{A}_{s}-\hat{E}_{11}^{-1} \hat{A}_{s}\right\| \leq N_{2}\left(M_{1}+M_{5}\right) \delta+N_{2}^{2} M_{5} \delta^{2}=: M_{6}(\delta) \delta$, 
where $M_{6}(\delta)=N_{2}\left(M_{1}+M_{3}+M_{3} M_{4}\right)+O(\delta)$.

These elementary calculations show that the explicit underlying ODE is only affected by a small perturbation if the perturbations $\Delta E, \Delta A$ affecting the DAE system are sufficiently small. Furthermore, since the boundedness condition (28) is fulfilled, the Sacker-Sell spectrum of the perturbed DAE system is exactly that of the associated underlying ODE system. This then implies the following stability result for the Sacker-Sell spectrum (and the resolvent set).

Theorem 58 Consider the DAE system (13) and the perturbed system (26). Assume that (28) holds and that (13) has a Sacker-Sell spectrum that consists of $k, k \leq d$, disjoint increasingly ordered closed finite intervals, $i$. e.,

$$
\Sigma_{S}=\bigcup_{i=1}^{k}\left[\alpha_{i}, \beta_{i}\right] .
$$

Let $\varepsilon>0$ be sufficiently small such that $\beta_{i}+\varepsilon<\alpha_{i+1}-\varepsilon$ for some $i, 0 \leq i \leq k$. For $i=0$ and $i=k$, set $\beta_{0}=-\infty$ and $\alpha_{k+1}=\infty$, respectively. Then there exists $\delta>0$ so that if the perturbations satisfy the inequality

$$
\max \left\{\sup _{t}\|\Delta \hat{E}(t)\|, \sup _{t}\|\Delta \hat{A}(t)\|\right\} \leq \delta,
$$

then the interval $\left(\beta_{i}+\varepsilon, \alpha_{i+1}-\varepsilon\right)$ is contained in the resolvent set of the perturbed DAE system (26).

Proof. Consider an arbitrary $\lambda \in\left(\beta_{i}+\varepsilon, \alpha_{i+1}-\varepsilon\right)$. Let the fundamental solution of the shifted system (32) be denoted by $\hat{X}_{\lambda}$ and the corresponding part for the underlying ODE by $\hat{X}_{1, \lambda}$. Since $\lambda$ belongs to the resolvent set of (13) and since $\lambda>\beta_{i}+\varepsilon$ and $\lambda<\alpha_{i+1}-\varepsilon$, it is clear that there exist a projection matrix $P$, and constants $\alpha, \beta>\varepsilon$ as well as $K, L \geq 1$ such that

$$
\begin{gathered}
\left\|\hat{X}_{1, \lambda}(t) P \hat{X}_{1, \lambda}^{-1}(s)\right\| \leq K e^{-\alpha(t-s)}, \quad t \geq s, \\
\left\|\hat{X}_{1, \lambda}(t)(I-P) \hat{X}_{1, \lambda}^{-1}(s)\right\| \leq L e^{\beta(t-s)}, \quad t \leq s .
\end{gathered}
$$

By invoking the Roughness Theorem (see [19, p. 34]) and using the estimate for the perturbation appearing in the associated explicit underlying ODE, if $\delta$ is sufficiently small such that in addition to the condition (44), the inequality

$$
\Delta=M_{6}(\delta) \delta<\min \left\{\frac{\alpha}{4 K^{2}}, \frac{\beta}{4 L^{2}}\right\},
$$

holds, then there exists a projection $\bar{P}$ with the same null-space as $P$ such that for the fundamental solution matrices $\bar{X}_{\lambda}$ and $\bar{X}_{1, \lambda}$ associated with the corresponding shifted system for (26) and its underlying shifted ODE, respectively, we have

$$
\begin{gathered}
\left\|\bar{X}_{1, \lambda}(t) \bar{P} \bar{X}_{1, \lambda}^{-1}(s)\right\| \leq \frac{5}{2} K^{2} e^{-(\alpha-2 K \Delta)(t-s)}, \quad t \geq s, \\
\left\|\bar{X}_{1, \lambda}(t)(I-\bar{P}) \bar{X}_{1, \lambda}^{-1}(s)\right\| \leq \frac{5}{2} L^{2} e^{(\beta-2 L \Delta)(t-s)}, \quad t \leq s .
\end{gathered}
$$

It is easy to see that by (45) it follows that $(\alpha-2 K \Delta)$ and $(\beta-2 L \Delta)$ are strictly positive. Thus, the perturbed and shifted underlying ODE has an exponential dichotomy. Applying Theorem 45, we obtain that the shifted and perturbed DAE system has an exponential dichotomy as well. But this means that $\lambda$ belongs to the resolvent set of the perturbed DAE system (26).

Corollary 59 Let the assumptions of Theorem 58 hold and let $\varepsilon>0$ be sufficiently small such that $\beta_{i-1}+\varepsilon<\alpha_{i}-\varepsilon<\alpha_{i} \leq \beta_{i}<\beta_{i}+\varepsilon<\alpha_{i+1}-\varepsilon$, for $0 \leq i \leq k$. For $i=0$ and $i=k$, set $\beta_{0}=-\infty$ and $\alpha_{k+1}=\infty$, respectively. Then, there exists $\delta>0$ so that if

$$
\max \left\{\sup _{t}\|\Delta \hat{E}(t)\|, \sup _{t}\|\Delta \hat{A}(t)\|\right\} \leq \delta,
$$


then under the effect of the perturbations, one or more new Sacker-Sell intervals may arise from the original Sacker-Sell interval $\left[\alpha_{i}, \beta_{i}\right]$, but this or these intervals are contained in the interval $\left[\alpha_{i}-\varepsilon, \beta_{i}+\varepsilon\right]$.

In this section we have analyzed the Lyapunov, and Sacker-Sell spectra for DAEs and their stability under perturbations. We have shown that the classical results for ODEs can be extended to DAEs. These results then form the basis for the computational methods that we consider in the following section.

\section{Numerical Computation of Spectral Intervals for DAEs}

In this section we extend the approaches that were derived for the computation of spectral intervals for ODEs in $[26,27,30]$ to DAEs. We derive numerical methods for computing Lyapunov and Sacker-Sell spectra for DAEs of the form (9) based on smooth $Q R$ factorizations. We discuss both continuous time and discrete time versions of these numerical methods.

\subsection{Continuous $Q R$-algorithm}

The basic idea for the numerical computation of spectral intervals for DAEs is to first transform the DAE system into an appropriate semi-implicit form, and then to apply a triangularization process to the coefficient matrices of the underlying implicit ODE. Throughout this section we assume that the DAE system is given in the strangeness-free form (9), i. e. whenever the value of $E(t), A(t)$ is needed, this has to be computed from the derivative array as described in Section 2. This can be done for example with the FORTRAN code GELDA [55] or the corresponding MATLAB version [56].

As we noted in Remark 13, although for the analysis we have preferred the semi-explicit form (13), in the numerical treatment we use the transformation from the strangeness-free system (9) to the form (14). The two systems (13) and (14) are globally kinematically equivalent, due to the results of Section 3 they have the same spectral intervals. Now suppose in addition that the lower row-block $A_{2}$ in (9) is continuously differentiable. By assumption it has fullrow rank. Therefore, see [21], there exist a nonsingular (and upper triangular) matrix function $\tilde{A}_{22} \in C^{1}\left(\mathbb{I}, \mathbb{R}^{(n-d) \times(n-d)}\right)$ and an orthogonal matrix function $\tilde{Q} \in C^{1}\left(\mathbb{I}, \mathbb{R}^{n \times n}\right)$ such that

$$
A_{2}=\left[\begin{array}{ll}
0 & \tilde{A}_{22}
\end{array}\right] \tilde{Q} .
$$

A numerical implementation of this smooth factorization can be obtained by using a sequence of Householder transformations applied to the augmented matrix

$$
\left[\begin{array}{l}
I_{n} \\
A_{2}
\end{array}\right]
$$

The triangularization process should be carried out pointwise from the bottom and the explicit multiplication of the elementary Householder transformations can be avoided. To make the factorization unique and to obtain the smoothness, we require the diagonal elements of $\tilde{A}_{22}$ to be positive, see [21]. Another possibility would be to derive differential equations for $\tilde{Q}$ (or its Householder factors) and to solve the corresponding initial value problems, see [21, 45].

The transformation $\tilde{x}=\tilde{Q}^{T} x$ leads to a DAE of the form (14), where

$$
\left[\begin{array}{cc}
\tilde{E}_{11} & \tilde{E}_{12} \\
0 & 0
\end{array}\right]=\left[\begin{array}{c}
E_{1} \\
0
\end{array}\right] \tilde{Q}, \quad\left[\begin{array}{cc}
\tilde{A}_{11} & \tilde{A}_{12} \\
0 & \tilde{A}_{22}
\end{array}\right]=\left[\begin{array}{c}
A_{1} \\
A_{2}
\end{array}\right] \tilde{Q}-\left[\begin{array}{c}
E_{1} \\
0
\end{array}\right] \dot{\tilde{Q}} .
$$

In order to evaluate $\dot{\tilde{Q}}$ at any time instance, we use either an appropriate finite difference formula or the method derived in [45]. 
Since in the form (14) the solution component $\tilde{x}_{2}$ associated with the algebraic equations vanishes, i. e., $\tilde{x}_{2}=0$, we only have to deal with the underlying implicit ODE (16) for the dynamic component $\tilde{x}_{1}$.

By the construction given in the proof of Lemma 55, there exist orthogonal matrix functions $U_{1} \in C\left(\mathbb{I}, \mathbb{R}^{n, n}\right)$ and $V_{1} \in C^{1}\left(\mathbb{I}, \mathbb{R}^{n, n}\right)$ such that the transformed matrix functions

$$
\mathcal{E}_{1}=\left[e_{i j}\right]=U_{1}^{T} \tilde{E}_{11} V_{1} \quad \text { and } \quad \mathcal{A}_{1}=\left[a_{i j}\right]=U_{1}^{T} \tilde{A}_{11} V_{1}-U_{1}^{T} \tilde{E}_{11} \dot{V}_{1}
$$

are both in upper triangular form. Combining this transformation with the preliminary change of variables $\tilde{x}=\tilde{Q}^{T} x$, we obtain that there exist orthogonal matrix functions $U=\operatorname{diag}\left(U_{1}, I_{a}\right) \in$ $C\left(\mathbb{I}, \mathbb{R}^{n \times n}\right), V=\tilde{Q} \operatorname{diag}\left(V_{1}, I_{a}\right) \in C^{1}\left(\mathbb{I}, \mathbb{R}^{n \times n}\right)$ such that by the new change of variables $z=V^{T} x$ and by multiplying both sides of $(9)$ with $U^{T}$ from the left, we arrive at a special upper triangular DAE system

$$
\left[\begin{array}{cc}
\mathcal{E}_{1} & U_{1}^{T} \tilde{E}_{12} \\
0 & 0
\end{array}\right] \dot{z}=\left[\begin{array}{cc}
\mathcal{A}_{1} & U_{1}^{T} \tilde{A}_{12} \\
0 & \tilde{A}_{22}
\end{array}\right] z,
$$

where $\mathcal{E}_{1}, \mathcal{A}_{1}$, and $\tilde{A}_{22}$ are upper triangular matrix functions of appropriate sizes.

In the case of explicit ODEs, i. e., if $E=I_{n}$, it is easy to see that $\tilde{Q}=I_{n}, U=V=U_{1}=V_{1}$ and the presented triangularization procedure reduces to that for ODEs in $[26,27]$.

In practice, we will need to evaluate only the diagonal elements of $\mathcal{A}_{1}$. As a consequence of the formula (40), by setting $K=\left[k_{i j}\right]=U_{1}^{T} \tilde{A}_{11} V_{1}$, we obtain

$$
a_{i i}=k_{i i}, \quad 1 \leq i \leq d
$$

Note also that it is not necessary to invert $\mathcal{E}_{1}$ in order to compute $S(Q)$ in (40). Indeed, let $L=\left[l_{i j}\right]$ be the strictly lower triangular part of $W_{1}=\mathcal{E}_{1}^{-1} U_{1}^{T} \tilde{A}_{11} V_{1}$, then (40) implies the linear system of equations

$$
\left[\begin{array}{cccc}
e_{1,1} & e_{1,2} & \cdots & e_{1, d} \\
0 & e_{2,2} & \cdots & e_{2, d} \\
\cdot & \cdot & \cdots & \cdot \\
0 & 0 & \cdots & e_{d, d}
\end{array}\right]\left[\begin{array}{cccc}
0 & 0 & \cdots & 0 \\
l_{2,1} & 0 & \cdots & 0 \\
\cdot & \cdot & \cdots & 0 \\
l_{d, 1} & l_{d, 2} & \cdots & 0
\end{array}\right]=\left[\begin{array}{cccc}
* & * & \cdots & * \\
k_{2,1} & * & \cdots & * \\
\cdot & \cdot & \cdots & * \\
k_{d, 1} & k_{d, 2} & \cdots & *
\end{array}\right]
$$

Solving for the entries $l_{i j}$ from the bottom row up to the top row we obtain

$$
\begin{array}{lll}
l_{d, j}=\frac{k_{d, j}}{e_{d, d}}, & j=1,2, \ldots, d-1, \\
l_{d-1, j}=\frac{k_{d-1, j}-e_{d-1, d} l_{d, j}}{e_{d-1, d-1}}, & j=1,2, \ldots, d-2, \\
l_{i, j}=\frac{k_{i, j}-\sum_{k=i+1}^{d} e_{i, k} l_{k, j}}{e_{i, i}}, & i=d-2, \ldots, 2 ; j=1, \ldots, i-1 .
\end{array}
$$

In this way the computational cost to determine $L$ and $S\left(V_{1}\right)$ is $d^{3} / 3+\mathcal{O}\left(d^{2}\right)$, only. The skewsymmetric matrix $S\left(V_{1}\right)$ is then given by

$$
S\left(V_{1}\right)=L-L^{T}
$$

Another important issue in the numerical implementation is to preserve the orthogonality of $V_{1}$ during the integration. There are different choices of methods to achieve this. The first is to use orthogonal integrators, e.g., Runge-Kutta-Gauß schemes, see [40]. The second is to apply first an arbitrary integration scheme, and then to reorthogonalize the obtained numerical solution at every grid point by a standard method, e. g., the Gram-Schmidt orthogonalization process. This is called projected integration. In this approach one may use, for instance, simple explicit Runge-Kutta methods like forward Euler and explicit trapezoidal methods [3] as we do in the numerical experiments presented in the next section. In the ODE case, in [25, 28], the authors have suggested and analyzed a third possibility, which is based on solving initial value problems for the elementary Householder or Givens transformations. An extension of the latter approach to implicit ODEs would give an efficient solution as well. We refer to [23, 27] for more details on the methods and numerical experiments in the case of explicit ODEs. 
Finally, we extend the procedures for computing the Lyapunov and Sacker-Sell spectral intervals to the implicit ODE of the form

$$
\mathcal{E}_{1}(t) \dot{R}_{1}=\mathcal{A}_{1}(t) R_{1}, \quad t \in \mathbb{I},
$$

with upper triangular matrix functions $\mathcal{E}_{1}, \mathcal{A}_{1}$. Here $R_{1}$ is a fundamental solution matrix of the triangularized underlying implicit ODE and it is exactly the $R$-part of a $Q R$-factorization of the fundamental solution to the underlying implicit ODE (16). By multiplying both sides of (51) by $\mathcal{E}_{1}^{-1}$, one arrives at an explicit ODE system of upper triangular form as in the ODE case. From the boundedness of $\tilde{E}_{11}^{-1} \tilde{A}_{11}$ and the proof of Lemma 55 , the boundedness of $\mathcal{E}_{1}^{-1} \mathcal{A}_{1}$ is obvious. However, the computation of $\mathcal{E}_{1}^{-1}$ should be avoided, because only the information lying in the diagonal elements is relevant for the computation of the spectral intervals.

In the following, we proceed as in the case of explicit ODEs in [27], where it has been shown that if the functions $a_{i i} / e_{i i}, i=1, \ldots, d$ are integrally separated, then the Lyapunov spectrum of the implicit ODE ( 51), which coincides with the Lyapunov spectrum of the DAE (9), can be determined as follows

$$
\Sigma_{L}=\bigcup_{i=1}^{d}\left[\lambda_{i}^{l}, \lambda_{i}^{u}\right]
$$

with

$$
\lambda_{i}^{l}:=\liminf _{t \rightarrow \infty} \frac{1}{t} \int_{0}^{t} \frac{a_{i i}(s)}{e_{i i}(s)} d s, \quad \lambda_{i}^{u}:=\limsup _{t \rightarrow \infty} \frac{1}{t} \int_{0}^{t} \frac{a_{i i}(s)}{e_{i i}(s)} d s, i=1,2, \ldots, d
$$

Let

$$
\lambda_{i}(t):=\frac{1}{t} \int_{0}^{t} \frac{a_{i i}(s)}{e_{i i}(s)} d s, \quad i=1,2, \ldots, d,
$$

which can be approximated by solving auxiliary initial value problems

$$
\begin{cases}\dot{\phi}_{i} & =\frac{a_{i i}(t)}{e_{i i}(t)}, \quad t \in \mathbb{I}, \quad i=1,2, \ldots, d \\ \phi_{i}(0) & =0\end{cases}
$$

and then setting

$$
\lambda_{i}(t)=\frac{1}{t} \phi_{i}(t), \quad i=1,2 \ldots, d
$$

Since

$$
\lambda_{i}^{l}=\lim _{\tau \rightarrow \infty} \inf _{t \geq \tau} \lambda_{i}(t) \quad \text { and } \lambda_{i}^{u}=\lim _{\tau \rightarrow \infty} \sup _{t \geq \tau} \lambda_{i}(t),
$$

for given $t_{0}$ and $T, 0<t_{0}<T, i=1,2, \ldots, d$, the quantities

$$
\lambda_{i}^{l}\left(t_{0}, T\right):=\inf _{t_{0} \leq t \leq T} \lambda_{i}(t) \quad \text { and } \lambda_{i}^{u}\left(t_{0}, T\right):=\sup _{t_{0} \leq t \leq T} \lambda_{i}(t)
$$

give approximate values for $\lambda_{i}^{l}$ and $\lambda_{i}^{u}$, respectively.

To test the integral separation of the functions $\left\{a_{i i} / e_{i i}\right\}_{i=1}^{d}$ in practice, we use a result of [1] that states that two scalar continuous functions $f_{1}, f_{2}$ are integrally separated if and only if there exists scalar $H>0$ such that their Steklov difference is positive, i.e., for $H$ sufficiently large, there exists $\beta>0$ such that

$$
f_{1}^{H}(t)-f_{2}^{H}(t) \geq \beta>0, \quad \text { for all } t \geq 0 .
$$

sufficiently large $H$, then the Steklov difference of $a_{i i} / e_{i i}$ and $a_{i+1, i+1} / e_{i+1, i+1}$ is given by

$$
S_{i}(t, H):=\frac{1}{H}\left\{\left[\phi_{i}(t+H)-\phi_{i}(t)\right]-\left[\phi_{i+1}(t+H)-\phi_{i+1}(t)\right]\right\}, t \in \mathbb{I}, i=1, \ldots, d-1 .
$$

Analogously, by the results of [31], the functions $a_{i i} / e_{i i}, i=1, \ldots, d$ also present information about the Sacker-Sell intervals of the implicit ODE (51) even without the integral separability. 
Concretely, for given $T \geq H>0$ and $0<t_{0}<T-H$, we set $b_{i i}(t)=a_{i i}(t) / e_{i i}(t), i=1,2, \ldots, d$, defined on $[0, T]$. We compute the Steklov averages of $b_{i i}$ with respect to the given $H$ as

$$
\psi_{i, H}(t):=\frac{1}{H} \int_{t}^{t+H} b_{i i}(s) d s, \quad T-H \geq t \geq t_{0} .
$$

This computation can be realized by solving auxiliary initial value problems as in the case of testing integral separation. Then, we use the quantities

$$
\kappa_{i}^{l}\left(t_{0}, T, H\right):=\inf _{t_{0} \leq t \leq T-H} \psi_{H, i}(t) \quad \text { and } \kappa_{i}^{u}\left(t_{0}, T, H\right):=\sup _{t_{0} \leq t \leq T-H} \psi_{H, i}(t)
$$

as approximations to the endpoints of the Sacker-Sell spectral intervals. Due to the property that the Sacker-Sell intervals include the Lyapunov intervals we then have obtain also bounds for the Lyapunov intervals.

We summarize the procedure for computing approximations to Lyapunov and Sacker-Sell spectral intervals in the following algorithm.

\section{Algorithm 1 (Continuous QR algorithm for computing Lyapunov and Sacker-Sell spectra)}

- Input: A pair of sufficiently matrix functions $(E, A)$ in the form of the strangeness-free DAE (9) (if they are not available directly they must be obtained pointwise as output of a routine such as GELDA); the values $T, H, \tau$ such that $H \in(0, T)$ and $\tau \in(0, T) ; V_{1}\left(t_{0}\right)$ as initial value for (41). Here we may use $V_{1}\left(t_{0}\right)=I_{d}$.

- Output: Bounds for spectral intervals $\left\{\lambda_{i}^{l}, \lambda_{i}^{u}\right\}_{i=1}^{d}$.

- Initialization:

1. Set $j=0, t_{0}:=0$. Compute $\tilde{Q}\left(t_{0}\right), \tilde{E}_{11}\left(t_{0}\right)$, and $\tilde{A}_{11}\left(t_{0}\right)$ as in (14).

2. Compute $U_{1}\left(t_{0}\right), \mathcal{E}_{1}\left(t_{0}\right), \mathcal{A}_{1}\left(t_{0}\right)$.

3. Set $\lambda_{i}\left(t_{0}\right)=0, \phi_{i}\left(t_{0}\right)=0, i=1, \ldots, d$.

While $t_{j}<T$

1. $j:=j+1$.

2. Choose a stepsize $h_{j}$ and set $t_{j}=t_{j-1}+h_{j}$.

3. Compute $\tilde{Q}\left(t_{j}\right)$, then $\tilde{E}_{11}\left(t_{j}\right), \tilde{A}_{11}\left(t_{j}\right)$, see (46) and (47).

4. Evaluate $V_{1}\left(t_{j}\right)$ by solving (41).

5. Compute $U_{1}\left(t_{j}\right), \mathcal{E}_{1}\left(t_{j}\right), \mathcal{A}_{1}\left(t_{j}\right)$ as in (42), (48), respectively.

6. Compute $\phi_{i}\left(t_{j}\right), \lambda_{i}\left(t_{j}\right), i=1, \ldots, d$ as in (54), (55).

7. Compute $S_{i}(t, H), i=1,2, \ldots, d-1$, by (56).

8. If desired, test integral separation via the Steklov difference.

9. Update $\min _{\tau \leq t \leq t_{j}} \lambda_{i}(t)$ and $\max _{\tau \leq t \leq t_{j}} \lambda_{i}(t)$.

The corresponding algorithm for computing Sacker-Sell spectra is similar. A slight difference is that instead of computing $\lambda_{i}(t)$ at each meshpoint (see Step 6.), we evaluate the Steklov averages $\psi_{H, i}(t)$ by the formula

$$
\psi_{H, i}(t)=\frac{1}{H}\left(\phi_{i}(t+H)-\phi_{i}(t)\right), \quad i=1,2, \ldots, d .
$$

Finally, we use the last step for computing $\inf _{\tau \leq t \leq T-H} \psi_{H, i}(t)$ and $\sup _{\tau \leq t \leq T-H} \psi_{H, i}(t)$. 


\section{$4.2 \quad$ Discrete $Q R$-algorithm}

While in the continuous $Q R$-algorithm, the fundamental solution matrix $R_{1}$ of the triangularized implicit ODE system (51) is not evaluated directly, in the discrete $Q R$-algorithm, $R_{1}$ is indirectly evaluated by a reorthogonalized integration of DAE system (9), an implicitly determined transformation to the semi-implicit form (14), and an appropriate $Q R$-factorization. Note that $R_{1}$ is upper triangular as well and the diagonal elements of the normalized $R_{1}$ are given by $e^{\phi_{i}(t)}, i=1,2, \ldots, d$, with the auxiliary functions $\phi_{i}$ defined in (54).

To apply the discrete $Q R$-algorithm, we first choose a mesh $0=t_{0}<t_{1}<\ldots<t_{N-1}<t_{N}=T$. (This mesh may be different from that in Algorithm 1). At $t_{0}$, we set

$$
Z_{0}=Q_{0}:=I_{d}
$$

For $j=1,2, \ldots, N$, let $X^{[j]}$ be the solution to the matrix initial value problem

$$
\left\{\begin{array}{l}
E \dot{X}^{[j]}=A X^{[j]}, \quad t_{j-1} \leq t \leq t_{j} \\
X^{[j]}\left(t_{j-1}\right)=\chi_{j-1},
\end{array}\right.
$$

with the initial condition

$$
\chi_{j-1}:=\tilde{Q}\left(t_{j-1}\right)\left[\begin{array}{c}
Q_{j-1} \\
0
\end{array}\right] .
$$

Here, $\tilde{Q}$ is defined and computed as in (46). We stress that $\chi_{j-1}$ defined in this way is a consistent initial value assigned at $t_{j-1}$ for DAE system (9). Then, we have that

$$
\tilde{Q}\left(t_{j}\right)^{T} X^{[j]}\left(t_{j}\right)=\left[\begin{array}{c}
Z_{j} \\
0
\end{array}\right]
$$

where $Z_{j}$ is the value of the rescaled fundamental solution matrix for the underlying ODE (16).

Then, we compute $Q R$-factorizations

$$
Z_{j}=Q_{j} \Theta_{j}, \quad j=1,2, \ldots, N
$$

where all the diagonal elements of the triangular matrices $\Theta_{j}$ are chosen to be positive. Now, letting $\tilde{X}_{1}$ be the normalized fundamental solution matrix of (16), then it follows that

$$
\begin{aligned}
\tilde{X}_{1}\left(t_{j}\right) & =Z_{j} Q_{j-1}^{T} \tilde{X}_{1}\left(t_{j-1}\right) \\
& =Q_{j} \Theta_{j} Q_{j-1}^{T} Q_{j-1} \Theta_{j-1} \cdots \Theta_{1} Q_{0} \\
& =Q_{j} \Theta_{j} \Theta_{j-1} \cdots \Theta_{1}
\end{aligned}
$$

Hence

$$
R_{1}\left(t_{j}\right)=\Theta_{j} \Theta_{j-1} \cdots \Theta_{1}
$$

Note that the quantities $\Theta_{j}$ give information about the local growth rates of the fundamental solution matrix $\tilde{X}_{1}$ on $\left[t_{j-1}, t_{j}\right]$. Furthermore, we obtain

$$
\lambda_{i}\left(t_{j}\right)=\frac{1}{t_{j}} \ln \left[R_{1}\left(t_{j}\right)\right]_{i, i}=\frac{1}{t_{j}} \ln \prod_{\ell=1}^{j}\left[\Theta_{\ell}\right]_{i, i}=\frac{1}{t_{j}} \sum_{\ell=1}^{j} \ln \left[\Theta_{\ell}\right]_{i, i}, \quad i=1,2, \ldots, d,
$$

where the functions $\lambda_{i}$ are defined as in (53). For computing the Lyapunov spectrum, we solve the associated optimization problems $\inf _{\tau \leq t \leq T} \lambda_{i}(t)$ and $\sup _{\tau \leq t \leq T} \lambda_{i}(t), i=1,2, \ldots, d$, respectively, with a given $\tau \in(0, T)$.

The approximation of the Sacker-Sell spectrum is obtained analogously. We summarize the procedure in the following algorithm.

\section{Algorithm 2 (Discrete $Q R$-algorithm for computing Lyapunov and Sacker-Sell spec- tra)}


- Input: A pair of sufficiently smooth matrix functions $(E, A)$ in the form of the strangeness-free DAE (9) (if they are not available directly they must be obtained pointwise as output of a routine such as GELDA), the time interval $[0, T], \tau \in(0, T)$, and a mesh $0=t_{0}<t_{1}<\ldots<t_{N-1}<t_{N}=T$

- Output: Bounds for spectral intervals $\left\{\lambda_{i}^{l}, \lambda_{i}^{u}\right\}_{i=1}^{d}$.

- Initialization:

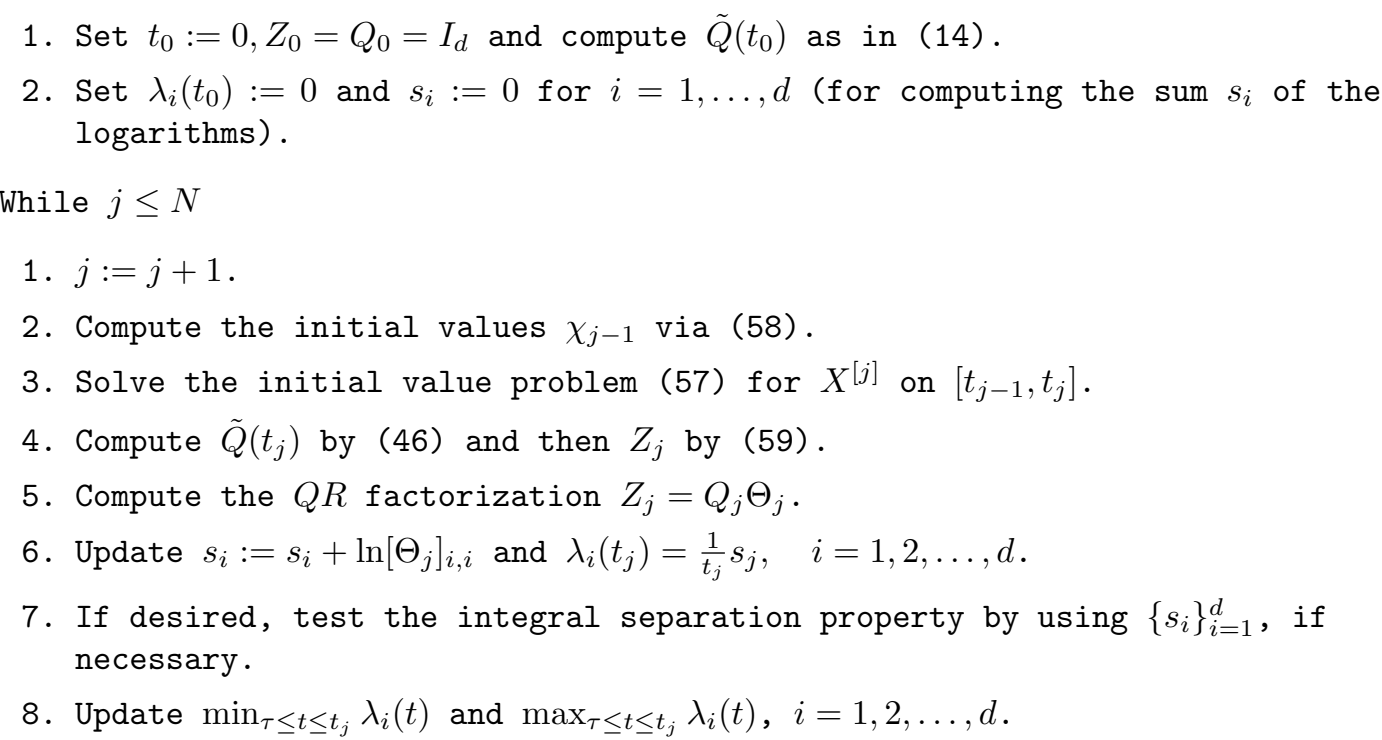

Remark 60 If the same mesh is used in Algorithms 1 and 2 and all calculations are done in exact arithmetic and without discretization errors, then the quantities $s_{i}$ at the end of the $j$-th step of Algorithm 2 are exactly the values $\phi_{i}\left(t_{j}\right)$ defined in Algorithm 1.

Advantages of the discrete algorithm are a simpler implementation and that existing DAE solvers for strangeness-free problems like BDF or implicit Runge-Kutta methods, see [3, 9, 41, 50] can be used. For example, in the numerical experiments presented in the next section, the backward Euler method is used. However, a disadvantage of the discrete method is that it creates numerical integration errors on each of the local intervals and these may grow very fast, in particular if the DAE system is very unstable and the subintervals are very long.

For non-regular systems, choosing sufficiently large bounds for $T, t_{0}$, and $H$ and giving error estimates for the approximate values of lim inf and limsup are difficult tasks and this is work in progress. For the illustration of some of these difficulties, see the numerical examples given in the next section. See also a brief discussion on error sources in [58].

\section{$5 \quad$ Numerical examples}

We have implemented both the continuous and the discrete variants of the QR methods described in Section 4 in MATLAB. The following results are obtained with Version 7.0 on an IBM computer with Intel CPU T2300 1.66 GHz. For the orthogonal integration, we have used the projected integration technique, see [22].

To illustrate the properties of the procedures we consider two examples, one of a Lyapunov regular DAE system and another DAE system which is not Lyapunov regular. In the second case, we calculated not only the Lyapunov spectral intervals, but also the Sacker-Sell intervals. 
Example 61 Our first example is a Lyapunov-regular DAE system which is constructed similar to the ODE examples in $[23,27]$. We derived a DAE system of the form (9) as follows. We began with an upper triangular implicit ODE system, applied appropriate transformations and then added additional algebraic variables. In this way we obtained a semi-implicit DAE system of the form (14) which was then transformed again to obtain a DAE system of the form (9) whose spectral information is the same as that of original implicit ODE system.

The original triangular implicit ODE system had the form $D(t) \dot{x}_{1}=B(t) \bar{x}_{1}$, where

$$
D(t)=\left[\begin{array}{cc}
1+\frac{1}{t+1} & 0 \\
0 & 1
\end{array}\right], \quad B(t)=\left[\begin{array}{cc}
\lambda_{1}-\frac{1}{t+1} & 1 \\
0 & \lambda_{2}+\cos (t+1)
\end{array}\right], \quad t \in \mathbb{I}, \quad \lambda_{i} \in \mathbb{R} \quad(i=1,2) .
$$

Here $\lambda_{i}, i=1,2,\left(\lambda_{1}<\lambda_{2}\right)$ are given real parameters. We then transformed and obtained the implicit ODE system $\tilde{E}_{11}(t) \dot{x}_{1}=\tilde{A}_{11}(t) \tilde{x}_{1}$ given by

$$
\tilde{E}_{11}=U_{1} D V_{1}^{T}, \quad \tilde{A}_{11}=U_{1} B V_{1}^{T}+U_{1} D V_{1}^{T} \dot{V}_{1} V_{1}^{T},
$$

with $U_{1}(t)=G_{\gamma_{1}}(t), V_{1}(t)=G_{\gamma_{2}}(t)$ with the Givens rotation

$$
G_{\gamma}(t)=\left[\begin{array}{cc}
\cos \gamma t & \sin \gamma t \\
-\sin \gamma t & \cos \gamma t
\end{array}\right]
$$

and some real parameters $\gamma_{1}, \gamma_{2}$. We chose additional blocks $\tilde{E}_{12}=U_{1}, \tilde{A}_{12}=V_{1}, \tilde{A}_{22}=U_{1} V_{1}$ and finally

$$
\tilde{E}=\left[\begin{array}{cc}
\tilde{E}_{11} & \tilde{E}_{12} \\
0 & 0
\end{array}\right], \quad \tilde{A}=\left[\begin{array}{cc}
\tilde{A}_{11} & \tilde{A}_{12} \\
0 & \tilde{A}_{22}
\end{array}\right]
$$

Using a $4 \times 4$ orthogonal matrix

$$
G(t)=\left[\begin{array}{cccc}
\cos \gamma_{3} t & 0 & 0 & \sin \gamma_{3} t \\
0 & \cos \gamma_{4} t & \sin \gamma_{4} t & 0 \\
0 & -\sin \gamma_{4} t & \cos \gamma_{4} t & 0 \\
-\sin \gamma_{3} t & 0 & 0 & \cos \gamma_{3} t
\end{array}\right]
$$

with real values $\gamma_{3}, \gamma_{4}$ we obtained $E=\tilde{E} G^{T}, A=A G^{T}+\tilde{E} G^{T} \dot{G} G^{T}$ and applied the methods to the DAE system $E(t) \dot{x}=A(t) x$ which is a strangeness-free DAE system of the form (9). Furthermore, because Lyapunov-regularity together with Lyapunov exponents are invariant with respect to orthogonal change of variables, this system is Lyapunov-regular with the Lyapunov exponents $\lambda_{1}, \lambda_{2}$.

For our numerical tests we have used the values

$$
\lambda_{1}=5, \lambda_{2}=0, \gamma_{1}=\gamma_{4}=2, \gamma_{2}=\gamma_{3}=1 .
$$

As numerical integration method in the continuous QR algorithm, we used the (projected) first order explicit Euler method and the (projected) second order explicit trapezoidal rule, both with constant stepsize $h$. The approximate values of the Lyapunov exponents are then calculated with different stepsizes $h$ and for different time intervals $[0, T]$. The results are displayed in Tables 1 and 2 , respectively. We display the CPU time measured in seconds.

The graph of the functions $\lambda_{1}(t), \lambda_{2}(t)$ is depicted in Figure 1. The monotonic, respectively oscillatory behavior of the two Lyapunov exponents is well approximated and the (admittedly slow) convergence of the computed Lyapunov exponents towards the exact Lyapunov exponents can be observed.

The numerical results of the discrete QR algorithm are displayed in Table 3. We have used the same meshes as in the computations with the continuous QR algorithm and we have used the implicit Euler method with a constant stepsize $h / 10$ for the numerical integration of the DAE in the subintervals. Without this refinement, the approximate values are substantially less accurate than the corresponding values computed by the continuous QR method. By comparing the numerical results for the continuous and discrete $\mathrm{QR}$ algorithm we see that the continuous QR method is more efficient and accurate than the discrete QR method. It is also interesting to observe that the discrete QR method oscillates when the stepsize is decreased. 


\begin{tabular}{|c|c|c|c|c|}
\hline$T$ & $h$ & $\lambda_{1}$ & $\lambda_{2}$ & $C P U-$ time \\
\hline 500 & 0.1 & 4.9341 & -0.0043 & 2.55 \\
\hline 500 & 0.05 & 4.9337 & -0.0038 & 5.01 \\
\hline 500 & 0.01 & 4.9337 & -0.0037 & 24.89 \\
\hline 1000 & 0.1 & 4.9632 & -0.0006 & 5.01 \\
\hline 1000 & 0.05 & 4.9628 & -0.0001 & 10.01 \\
\hline 1000 & 0.01 & 4.9627 & -0.0001 & 49.84 \\
\hline 2000 & 0.1 & 4.9799 & -0.0010 & 10.17 \\
\hline 2000 & 0.05 & 4.9794 & -0.0005 & 20.02 \\
\hline 10000 & 0.1 & 4.9956 & -0.0009 & 49.91 \\
\hline 10000 & 0.05 & 4.9951 & -0.0003 & 99.71 \\
\hline
\end{tabular}

Table 1: Lyapunov exponents for Example 61 computed via the continuous QR-Euler method

\begin{tabular}{|c|c|c|c|c|}
\hline$T$ & $h$ & $\lambda_{1}$ & $\lambda_{2}$ & $C P U-$ time \\
\hline 500 & 0.1 & 4.9333 & -0.0033 & 4.95 \\
\hline 500 & 0.05 & 4.9336 & -0.0036 & 9.83 \\
\hline 500 & 0.01 & 4.9337 & -0.0037 & 48.81 \\
\hline 1000 & 0.1 & 4.9624 & -0.0004 & 9.88 \\
\hline 1000 & 0.05 & 4.9626 & -0.0002 & 19.61 \\
\hline 1000 & 0.01 & 4.9951 & -0.0003 & 100.28 \\
\hline 2000 & 0.1 & 4.9789 & 0.0000 & 19.63 \\
\hline 2000 & 0.05 & 4.9951 & -0.0003 & 101.02 \\
\hline 10000 & 0.1 & 4.9946 & 0.0002 & 97.55 \\
\hline 10000 & 0.05 & 4.9948 & -0.0001 & 195.51 \\
\hline
\end{tabular}

Table 2: Lyapunov exponents for Example 61 computed via the continuous QR-Trapezoid method

Figure 1: Graph of the functions $\lambda_{i}(t), i=1,2$ in Example 61. 


\begin{tabular}{|c|c|c|c|c|}
\hline$T$ & $h$ & $\lambda_{1}$ & $\lambda_{2}$ & $C P U-$ time \\
\hline 500 & 0.1 & 5.0324 & -0.0137 & 9.87 \\
\hline 500 & 0.05 & 4.9818 & -0.0087 & 19.59 \\
\hline 500 & 0.01 & 4.9431 & -0.0047 & 97.31 \\
\hline 1000 & 0.1 & 5.0625 & -0.0100 & 19.63 \\
\hline 1000 & 0.05 & 5.0114 & -0.0050 & 38.87 \\
\hline 2000 & 0.1 & 5.0799 & -0.0104 & 39.20 \\
\hline 2000 & 0.05 & 5.0284 & -0.0053 & 78.15 \\
\hline 10000 & 0.1 & 5.0963 & -0.0102 & 194.89 \\
\hline 10000 & 0.05 & 5.0443 & -0.0052 & 389.64 \\
\hline
\end{tabular}

Table 3: Lyapunov exponents for Example 61 computed by the discrete QR method with the implicit Euler method as integrator

\begin{tabular}{|c|c|c|c|c|c|}
\hline$T$ & $t_{0}$ & $h$ & {$\left[\lambda_{1}^{l}, \lambda_{1}^{u}\right]$} & {$\left[\lambda_{2}^{l}, \lambda_{2}^{u}\right]$} & $C P U-$ time \\
\hline 1000 & 100 & 0.1 & {$[-1.0018,0.5865]$} & {$[-6.0006,-4.8928]$} & 5.28 \\
\hline 5000 & 100 & 0.1 & {$[-1.0018,1.0004]$} & {$[-6.0006,-4.3846]$} & 26.02 \\
\hline 10000 & 100 & 0.1 & {$[-1.0018,1.0004]$} & {$[-6.0006,-4.0235]$} & 51.52 \\
\hline 10000 & 500 & 0.1 & {$[-0.0647,1.0004]$} & {$[-6.0006,-4.0235]$} & 51.63 \\
\hline 10000 & 100 & 0.05 & {$[-1.0028,1.0000]$} & {$[-6.0001,-4.0229]$} & 103.42 \\
\hline 20000 & 100 & 0.1 & {$[-1.0018,1.0004]$} & {$[-6.0006,-4.0007]$} & 103.50 \\
\hline 20000 & 500 & 0.1 & {$[-0.4598,1.0004]$} & {$[-6.0006,-4.0007]$} & 103.32 \\
\hline 20000 & 100 & 0.05 & {$[-1.0028,1.0000]$} & {$[-6.0001,-4.0001]$} & 210.95 \\
\hline 50000 & 100 & 0.05 & {$[-1.0028,1.0000]$} & {$[-6.0001,-4.0001]$} & 519.45 \\
\hline 50000 & 500 & 0.05 & {$[-0.9844,1.0000]$} & {$[-6.0001,-4.0001]$} & 518.15 \\
\hline 100000 & 100 & 0.05 & {$[-1.0028,1.0000]$} & {$[-6.0001,-4.0001]$} & 1044.94 \\
\hline 100000 & 500 & 0.05 & {$[-0.9998,1.0000]$} & {$[-6.0001,-4.0001]$} & 1050.36 \\
\hline
\end{tabular}

Table 4: Lyapunov spectral intervals for Example 62 computed by the continuous QR-Euler method

Example 62 (A DAE system which is not Lyapunov regular) With the same transformations as in Example 61 we also constructed a DAE that is not Lyapunov regular by changing the matrix $B(t)$ in Example 61 to

$$
B(t)=\left[\begin{array}{cc}
\sin (\ln (t+1))+\cos (\ln (t+1))+\lambda_{1} & 1 \\
0 & \sin (\ln (t+1))-\cos (\ln (t+1))+\lambda_{2}
\end{array}\right], \quad t \in \mathbb{I} .
$$

Here we chose $\lambda_{1}=0, \lambda_{2}=-5$. Since Lyapunov and Sacker-Sell spectra are invariant with respect to global kinematical equivalence transformation, it is easy to compute the Lyapunov spectral intervals as $[-1,1]$ and $[-6,-4]$ and the Sacker-Sell spectral intervals as $[-\sqrt{2}, \sqrt{2}]$ and $[-5-\sqrt{2},-5+\sqrt{2}]$.

We computed first the approximate Lyapunov spectral intervals with different initial and end points $t_{0}, T$, and stepsizes $h$ via the continuous QR-Euler method. The results are displayed in Table 4 and we observe that the method computes reasonably good approximations to the Lyapunov spectral intervals but that the method is sensitive to the choice of the values of $T$ and $t_{0}$. This is already a well-known difficulty in the case of ODEs, see [27]. The graphs of the functions $\lambda_{1}(t), \lambda_{2}(t)$ are shown in Figure 2.

Finally, we used the continuous QR algorithm for approximating the Sacker-Sell intervals with different $T, H$ and $h$. The numerical results displayed in Table 5 illustrate well the success of the 
Figure 2: The graph of functions $\lambda_{i}(t), i=1,2$ in Example 62 .

\begin{tabular}{|c|c|c|c|c|c|}
\hline$T$ & $H$ & $h$ & {$\left[\kappa_{1}^{l}, \kappa_{1}^{u}\right]$} & {$\left[\kappa_{2}^{l}, \kappa_{2}^{u}\right]$} & $C P U-$ time \\
\hline 1000 & 100 & 0.1 & {$[-1.2042,1.3811]$} & {$[-6.4049,-4.8927]$} & 6.20 \\
\hline 5000 & 100 & 0.1 & {$[-1.2042,1.4131]$} & {$[-6.4049,-3.5990]$} & 30.79 \\
\hline 10000 & 100 & 0.1 & {$[-1.2042,1.4131]$} & {$[-6.4049,-3.5867]$} & 61.94 \\
\hline 10000 & 500 & 0.1 & {$[-0.7327,1.4030]$} & {$[-6.2142,-3.5872]$} & 94.80 \\
\hline 10000 & 100 & 0.05 & {$[-1.2049,1.4127]$} & {$[-6.4046,-3.5860]$} & 147.19 \\
\hline 20000 & 100 & 0.1 & {$[-1.3461,1.4131]$} & {$[-6.4049,-3.5867]$} & 123.57 \\
\hline 20000 & 500 & 0.1 & {$[-1.3416,1.4030]$} & {$[-6.2142,-3.5872]$} & 201.26 \\
\hline 20000 & 100 & 0.05 & {$[-1.3468,1.4127]$} & {$[-6.4046,-3.5860]$} & 283.10 \\
\hline 50000 & 100 & 0.1 & {$[-1.4132,1.4131]$} & {$[-6.4049,-3.5867]$} & 310.36 \\
\hline 50000 & 500 & 0.1 & {$[-1.4132,1.4030]$} & {$[-6.2142,-3.5872]$} & 506.65 \\
\hline 100000 & 100 & 0.1 & {$[-1.4132,1.4131]$} & {$[-6.4049,-3.5867]$} & 646.15 \\
\hline 100000 & 500 & 0.1 & {$[-1.4132,1.4030]$} & {$[-6.3633,-3.5872]$} & 976.30 \\
\hline 200000 & 500 & 0.1 & {$[-1.4132,1.4030]$} & {$[-6.4147,-3.5872]$} & 1973.43 \\
\hline
\end{tabular}

Table 5: Sacker-Sell spectral intervals for Example 62 computed by the continuous QR-Euler method 
Figure 3: Graph of Steklov averages $\psi_{H, i}(t), i=1,2, H=500$ in Example 62.

QR algorithm but also the difficulty in choosing appropriately large values of $T$ and $H$. A plot of the graph of the Steklov averages $\psi_{H, i}(t), i=1,2$ with $H=500$ is given in Figure 3.

In order to improve the described numerical methods it is important to carry out a careful error analysis of the different components of the method as well as a detailed analysis of the convergence behavior with respect to the choice of initial and end point $t_{0}, T$. This is current work in progress.

\section{Conclusion}

In this paper we have extended the classical spectral concepts and numerical methods for approximating (Lyapunov, Bohl and Sacker-Sell) spectral intervals that are well-known for ordinary differential equations to linear differential-algebraic equations with variable coefficients.

In the theoretical analysis of the spectral theory we have used appropriate orthogonal changes of variables to transform the original DAE system to a particular strangeness-free form for which the underlying ODE systems are easily obtained. The relationship between different spectra of the DAE systems and those of their corresponding underlying ODE system has been analyzed. We have proven that under some boundedness conditions, the Lyapunov and the Sacker-Sell (exponential dichotomy) spectrum of a DAE system and those of its underlying ODE system coincide. Several significant differences between the spectral theory for ODEs and that for DAEs have been discussed as well and the stability of these spectra has been investigated. In particular, we have shown that the Sacker-Sell spectrum of a robustly strangeness-free DAE system is stable with respect to admissible structured perturbations. In general, if either the DAE system under consideration is not robustly strangeness-free or it is subject to an unstructured perturbation, then the spectral stability cannot be expected. We have proposed two numerical methods based on QR factorization for calculating Lyapunov and Sacker-Sell spectra. The algorithms as well as related implementation techniques have been discussed. Finally, two DAE examples have been presented for illustration.

Experimental numerical results have not only illustrated the efficiency and the reliability of the computational methods, but the numerical results also indicate the difficulties that may arise in the implementation and the use of these methods. In particular a detailed error and perturbation analysis is necessary. Similarly to the ODE case, an extension of such algorithms to nonlinear DAEs should also be carried out. 
Further work is also necessary in developing more efficient implementation techniques and a complete error analysis for the overall numerical methods proposed in this paper.

\section{Acknowledgment}

We thank E. Van Vleck for interesting discussions and bringing the concept of Sacker-Sell spectra and their numerical computation to our attention. We also thank A. Ilchmann for providing a copy of the original paper of Bohl. Last but not least, we thank an anonymous referee for his(her) useful comments and suggestions that led to this improved version of the paper.

\section{References}

[1] L. Ya. Adrianova. Introduction to linear systems of differential equations. Trans. Math. Monographs, Vol. 146, AMS, Providence, RI, 1995.

[2] U. M. Ascher and L. R. Petzold. Stability of computation for constrained dynamical systems. SIAM J. Sci. Statist. Comput., 14:95-120, 1993.

[3] U. M. Ascher and L. R. Petzold. Computer Methods for Ordinary Differential Equations and Differential-Algebraic Equations. Society for Industrial and Applied Mathematics (SIAM), Philadelphia, PA., 1998.

[4] K. Balla and V. H. Linh. Adjoint pairs of differential-algebraic equations and Hamiltonian systems. Appl. Numer. Math., 53:131-148, 2005.

[5] K. Balla and R. März. Linear differential algebraic equations of index 1 and their adjoint equations. Res. in Math., 37:13-35, 2000.

[6] K. Balla and R. März. A unified approach to linear differential algebraic equations and their adjoints. Z. Anal. Anwendungen, 21:783-802, 2002.

[7] P. Bohl. Über Differentialungleichungen. J. f. d. Reine und Angew. Math., 144:284-313, 1913.

[8] K. E. Brenan, S. L. Campbell, and L. R. Petzold. The Numerical Solution of Initial-Value Problems in Ordinary Differential-Algebraic Equations. Elsevier, North Holland, New York, N. Y., 1989.

[9] K. E. Brenan, S. L. Campbell, and L. R. Petzold. Numerical Solution of Initial-Value Problems in Differential Algebraic Equations. SIAM Publications, Philadelphia, PA, 2nd edition, 1996.

[10] R. Byers and N.K. Nichols. On the stability radius of a generalized state-space system. Lin. Alg. Appl., 188-189:113-134, 1993.

[11] S. L. Campbell. Comment on controlling generalized state-space (descriptor) systems. Internat. J. Control, 46:2229-2230, 1987.

[12] S. L. Campbell. Linearization of DAE's along trajectories. Z. Angew. Math. Phys., 46:70-84, 1995.

[13] S. L. Campbell and C. W. Gear. The index of general nonlinear DAEs. Numer. Math., 72:173-196, 1995.

[14] S. L. Campbell, N. K. Nichols, and W. J. Terrell. Duality, observability, and controllability for linear time-varying descriptor systems. Circ. Syst. Signal Process., 10:455-470, 1991.

[15] J.-L. Chern and L. Dieci. Smoothness and periodicity of some matrix decompositions. SIAM J. Matr. Anal. Appl., 22:772-792, 2000. 
[16] C.J. Chyan, N.H. Du, and V.H. Linh. On data-dependence of exponential stability and the stability radii for linear time-varying differential-algebraic systems. J. Diff. Equations, 245:2078-2102, 2008.

[17] N.D. Cong and H. Nam. Lyapunov's inequality for linear differential algebraic equation. Acta Math. Vietnam, 28:73-88, 2003.

[18] N.D. Cong and H. Nam. Lyapunov regularity of linear differential algebraic equations of index 1. Acta Math. Vietnam, 29:1-21, 2004.

[19] W. A. Coppel. Dichotomies in Stability Theory. Springer-Verlag, New York, NY, 1978.

[20] J.L. Daleckii and M.G. Krein. Stability of solutions of differential equations in Banach spaces. American Mathematical Society, Providence, RI, 1974.

[21] L. Dieci and T. Eirola. On smooth decompositions of matrices. SIAM J. Matr. Anal. Appl., 20:800-819, 1999.

[22] L. Dieci, R. D. Russell, and E. S. Van Vleck. Unitary integrators and applications to continuous orthonormalization techniques. SIAM J. Numer. Anal., 31:261-281, 1994.

[23] L. Dieci, R. D. Russell, and E. S. Van Vleck. On the computation of Lyapunov exponents for continuous dynamical systems. SIAM J. Numer. Anal., 34:402-423, 1997.

[24] L. Dieci and E. S. Van Vleck. Computation of a few Lyapunov exponents for continuous and discrete dynamical systems. Appl. Numer. Math., 17:275-291, 1995.

[25] L. Dieci and E. S. Van Vleck. Computation of orthonormal factors for fundamental solution matrices. Numer. Math., 83:599-620, 1999.

[26] L. Dieci and E. S. Van Vleck. Lyapunov and other spectra: a survey. In Collected lectures on the preservation of stability under discretization (Fort Collins, CO, 2001), pages 197-218. SIAM, Philadelphia, PA, 2002.

[27] L. Dieci and E. S. Van Vleck. Lyapunov spectral intervals: theory and computation. SIAM J. Numer. Anal., 40:516-542, 2002.

[28] L. Dieci and E. S. Van Vleck. Orthonormal integrators based on Householder and Givens transformations. Future Generation Computer Systems, 19:363-373, 2003.

[29] L. Dieci and E. S. Van Vleck. On the error in computing Lyapunov exponents by QR methods. Numer. Math., 101:619-642, 2005.

[30] L. Dieci and E. S. Van Vleck. Lyapunov and Sacker-Sell spectral intervals. J. Dyn. Diff. Eq., 19:265-293, 2006.

[31] L. Dieci and E. S. Van Vleck. Perturbation theory for approximation of Lyapunov exponents by QR methods. J. Dyn. Diff. Eq., 18:815-842, 2006.

[32] M. Diehl, D. B. Leineweber, A. Schäfer, H. G. Bock, and J. P. Schlöder. Optimization of multiple-fraction batch distillation with recycled waste cuts. AIChE Journal, 48(12):2869$2874,2002$.

[33] M. Diehl, I. Uslu, R. Findeisen, S. Schwarzkopf, F. Allgöwer, H. G. Bock, T. Bürner, E. D. Gilles, A. Kienle, J. P. Schlöder, and E. Stein. Real-time optimization for large scale processes: Nonlinear model predictive control of a high purity distillation column. In M. Grötschel, S. O. Krumke, and J. Rambau, editors, Online Optimization of Large Scale Systems: State of the Art, pages 363-384. Springer, 2001. 
[34] N.H. Du and V.H. Linh. Robust stability of implicit linear systems containing a small parameter in the leading term. IMA J. Math. Cont. Inf., 23:67-84, 2006.

[35] N.H. Du and V.H. Linh. Stability radii for linear time-varying differential-algebraic equations with respect to dynamic perturbations. J. Diff. Equations, 230:579-599, 2006.

[36] E. Eich-Soellner and C. Führer. Numerical Methods in Multibody Systems. Teubner Verlag, Stuttgart, Germany, 1998.

[37] E. Griepentrog and R. März. Differential-Algebraic Equations and their Numerical Treatment. Teubner Verlag, Leipzig, Germany, 1986.

[38] M. Günther and U. Feldmann. CAD-based electric-circuit modeling in industry I. Mathematical structure and index of network equations. Surv. Math. Ind., 8:97-129, 1999.

[39] M. Günther and U. Feldmann. CAD-based electric-circuit modeling in industry II. Impact of circuit configurations and parameters. Surv. Math. Ind., 8:131-157, 1999.

[40] E. Hairer, C. Lubich, and G. Wanner. Geometric Numerical Integration. Structure-Preserving Algorithms for Ordinary Differential Equations. Springer-Verlag, Berlin, Germany, 2002.

[41] E. Hairer and G. Wanner. Solving Ordinary Differential Equations II: Stiff and DifferentialAlgebraic Problems. Springer-Verlag, Berlin, Germany, 2nd edition, 1996.

[42] I. Higueras, R. März, and C. Tischendorf. Stability preserving integration of index-1 DAEs. Appl. Numer. Math., 45:175-200, 2003.

[43] I. Higueras, R. März, and C. Tischendorf. Stability preserving integration of index-2 DAEs. Appl. Numer. Math., 45:201-229, 2003.

[44] D. Hinrichsen and A. J. Pritchard. Mathematical Systems Theory I. Modelling, State Space Analysis, Stability and Robustness. Springer-Verlag, New York, NY, 2005.

[45] P. Kunkel and V. Mehrmann. Smooth factorizations of matrix valued functions and their derivatives. Numer. Math., 60:115-132, 1991.

[46] P. Kunkel and V. Mehrmann. Canonical forms for linear differential-algebraic equations with variable coefficients. J. Comput. Appl. Math., 56:225-259, 1994.

[47] P. Kunkel and V. Mehrmann. Generalized inverses of differential-algebraic operators. SIAM J. Matr. Anal. Appl., 17:426-442, 1996.

[48] P. Kunkel and V. Mehrmann. Regular solutions of nonlinear differential-algebraic equations and their numerical determination. Numer. Math., 79:581-600, 1998.

[49] P. Kunkel and V. Mehrmann. Analysis of over- and underdetermined nonlinear differentialalgebraic systems with application to nonlinear control problems. Math. Control, Signals, Sys., 14:233-256, 2001.

[50] P. Kunkel and V. Mehrmann. Differential-Algebraic Equations. Analysis and Numerical Solution. EMS Publishing House, Zürich, Switzerland, 2006.

[51] P. Kunkel and V. Mehrmann. Optimal control for unstructured nonlinear differential-algebraic equations of arbitrary index. To appear in Mathematics of Control Signals and Systems, 2008.

[52] P. Kunkel and V. Mehrmann. Optimal control for linear descriptor systems with variable coefficients Proceedings of the IEEE Conference NLASSC 2007, 9.-11.1.07 Kharagpur, India.

[53] P. Kunkel and V. Mehrmann. Stability properties of differential-algebraic equations and spin-stabilized discretization. Electr. Trans. Num. Anal., 26:385-420, 2007. 
[54] P. Kunkel, V. Mehrmann, and W. Rath. Analysis and numerical solution of control problems in descriptor form. Math. Control, Signals, Sys., 14:29-61, 2001.

[55] P. Kunkel, V. Mehrmann, W. Rath, and J. Weickert. A new software package for linear differential-algebraic equations. SIAM J. Sci. Comput., 18:115-138, 1997.

[56] P. Kunkel, V. Mehrmann, and S. Seidel. A MATLAB package for the numerical solution of general nonlinear differential-algebraic equations. Technical Report 16/2005, Institut für Mathematik, TU Berlin, Berlin, Germany, 2005. url: http://www.math.tu-berlin.de/preprints/.

[57] M. Lentini and R. März. Conditioning and dichotomy in differential algebraic equations. SIAM J. Numer. Anal., 27:1519-1526, 1990.

[58] V.H. Linh and V. Mehrmann. Spectral intervals for differential algebraic equations and their numerical approximations. Preprint 402, DFG Research Center MAThEOn, TU Berlin, Berlin, Germany, 2007. url: http://www.matheon.de/ .

[59] A. M. Lyapunov. The general problem of the stability of motion. Translated by A. T. Fuller from Edouard Davaux's French translation (1907) of the 1892 Russian original. Internat. J. Control, pages 521-790, 1992.

[60] R. März. Criteria for the trivial solution of differential algebraic equations with small nonlinearities to be asymptotically stable. J. Math. Anal. Appl., 225:587-607, 1998.

[61] R. März. The index of linear differential algebraic equations with properly stated leading terms. Res. in Math., 42:308-338, 2002.

[62] R. März and A. R. Rodriguez-Santiesteban. Analyzing the stability behaviour of solutions and their approximations in case of index-2 differential-algebraic systems. Math. Comp., 71:605-632, 2001.

[63] R. M. M. Mattheij and P. M. E. J. Wijckmans. Sensitivity of solutions of linear DAE to perturbations of the system matrices. Numer. Alg., 19:159-171, 1998.

[64] M. Otter, H. Elmqvist, and S. E. Mattson. Multi-domain modeling with modelica. In Paul Fishwick, editor, CRC Handbook of Dynamic System Modeling. CRC Press, 2006. To appear.

[65] O. Perron. Die Ordnungszahlen Linearer Differentialgleichungssysteme. Math. Zeits., 31:748$766,1930$.

[66] P. J. Rabier and W. C. Rheinboldt. Theoretical and Numerical Analysis of DifferentialAlgebraic Equations, volume VIII of Handbook of Numerical Analysis. Elsevier Publications, Amsterdam, The Netherlands, 2002.

[67] W. C. Rheinboldt. Differential-algebraic systems as differential equations on manifolds. Math. Comp., 43:473-482, 1984.

[68] R. Riaza. Stability issues in regular and non-critical singular DAEs. Acta Appl. Math., $73: 243-261,2002$.

[69] R. Riaza and C. Tischendorf. Topological analysis of qualitative features in electrical circuit theory. Technical Report 04-18, Institut für Mathematik, Humboldt Universität zu Berlin, Berlin, Germany, 2004.

[70] R.J. Sacker and G.R. Sell. A spectral theory for linear differential systems. J. Diff. Equations, 27:320-358, 1978.

[71] T. Stykel. Analysis and Numerical Solution of Generalized Lyapunov Equations. Dissertation, Institut für Mathematik, TU Berlin, Berlin, Germany, 2002. 
[72] T. Stykel. On criteria for asymptotic stability of differential-algebraic equations. Z. Angew. Math. Mech., 92:147-158, 2002.

[73] T. Stykel. Stability and inertia theorems for generalized Lyapunov equations. Lin. Alg. Appl., 355:297-314, 2002.

[74] C. Tischendorf. On stability of solutions of autonomous index-1 tractable and quasilinear index-2 tractable DAE's. Circ. Syst. Signal Process., 13:139-154, 1994. 